Kragujevac Journal of Mathematics

Volume 40(2) (2016), Pages 194-222.

\title{
BERGMAN-TYPE INTEGRAL OPERATORS ON SEMIPRODUCTS OF THE TUBE DOMAINS OVER SYMMETRIC CONES AND MULTIFUNCTIONAL ANALYTIC SPACES
}

\author{
S. KURILENKO ${ }^{1}$ AND R. SHAMOYAN ${ }^{1}$
}

\begin{abstract}
We introduce new Bergman type operators in semiproducts of tubular domains over symmetric cones. Properties of such Bergman-type integral operators on semi-products of the tubular domains over symmetric cones are obtained. We also define new multifunctional analytic spaces in tubular domains over symmetric cones and provide their basic properties.
\end{abstract}

\section{INTRODUCTION}

The goal of this paper is to provide complete analogues of our previous results from $[17,27]$ in the unit disk and unit ball in context of more complicated tubular domains over symmetric cones.

This paper consists of two separate parts. The first part is devoted to new assertions on integral Bergman-type operators acting on some new Bergman type spaces in products (or semi-products) of tubular domains over symmetric cones and the second one with related new analytic multifunctional spaces in tubular domains over symmetric cones. Previously it was noted these two topics are related in simpler domains in the unit disk and in the unit ball (see [17,26,27]). Parallel assertions are also valid in context of general bounded strictly pseudoconvex domains with smooth boundary (see $[29,32])$. To summarize this paper and [29,32] show in most typical Siegel domains such as bounded strongly pseudonconvex domains with smooth boundary and unbounded tubular domains over symmetric cones main assertions from [17,27] are also valid. It is a fundamental fact of the theory of analytic Bergman function

Key words and phrases. Bergman-type integral operators, tubular domains, analytic functions, Bergman-type spaces, multifunctional analytic spaces in tubular domains.

2010 Mathematics Subject Classification. Primary: 32A07. Secondary: 32A10, 32A37.

Received: December 3, 2014.

Accepted: December 15, 2015. 
spaces in the unit disk that the Bergman projection is a bounded operator in analytic Bergman $A_{\alpha}^{p}$ spaces, $1<p<\infty, \alpha>-1$ in the unit disk $D$ (see [12,21,25,33]). This fact alone has many application in function theory of one complex variables, and also in operator theory and related areas. Various analogues and extensions of this result to various domains in one and higher dimension and various related function spaces in one and higher dimension and their applications are also well-known in literature. We refer the reader again to $[12,21,25,33]$ and to various references there. To formulate this basic result in the unit disk we need several basic definitions.

Let $D$ be a unit disk in complex plane $\mathbb{C}$. Let $H(D)$ be the spaces of all analytic functions in the unit disk.

Then let

$A_{p}^{\alpha}(D)=\left\{f \in H(D): \int_{D}|f(z)|^{p}(1-|z|)^{\alpha} d m_{2}(z)<\infty\right\}, \quad$ for $1<p<\infty, \alpha>-1$

be the classical Bergman space in the unit disk. Replacing as usual $A$ by $L$ we get the corresponding class of measurable functions in the unit disk. We denote as usual by $d m_{2}$ the Lebeques measure in the unit disk $D$. These spaces are Banach spaces.

Theorem 1.1 (see [12]). The Bergman projection

$$
T_{\beta}(F)(z)=\int_{D} Q_{\beta}(z, w) F(w)(1-|w|)^{\beta} d m_{2}(w),
$$

is a bounded operator from $L_{\alpha}^{p}$ to $A_{\alpha}^{p}$ and moreover the following estimate is valid.

$$
\left\|\int_{D} Q_{\beta}(z, w) F(w)(1-|w|)^{\beta} d m_{2}(w)\right\|_{A_{\alpha}^{p}} \leq c\|F\|_{L_{\alpha}^{p}}
$$

where $\beta>\frac{1+\alpha}{p}-1, \alpha>-1,1<p<\infty, Q_{\alpha}(z, w)=\frac{(1+\alpha)}{\pi(1-z \bar{w})^{2+\alpha}}, z, w \in D$.

A complete analogue of this theorem is valid in simplest high-dimensional domains in $\mathbb{C}^{n}$ namely in the unit ball and polydisk (see, for example, [12,21,22, 25,33] and various references there). They also serve as a base of many assertions in function theory, but of several complex variables (in the unit polydisk and in the unit ball).

The intention of this paper is to search new complete extensions of Theorem 1.1 in more difficult unbounded domains in higher dimension namely in tubular domains over symmetric cones in $\mathbb{C}^{n}$. These issues were under intensive study during last decade (see, for example, $[2,4,23,24,30]$ and references there). Note also to find applications of such type theorems in tubular domain over symmetric cones is also an important issue and various aspects of this topic can be seen in recent papers $[2,4,23,24,30]$. Our work on the other hand is also related to issues related to product or semiproduct domains. The product domains are very vital simply since they are the simplest examples of domains with non-smooth boundaries. Recently many papers appeared in this direction. As example we mention that in [11] and [10] authors recently studied the existence and regularity of the well-known d-problem (equation) on product domains. 
We refer the reader also to [9] for some recent results on function spaces on product domains related with studies of regularity of the d-problem using various techniques. In particular various embeddings of function spaces on product domains have important applications in [11] and [10]. Bergman type integral operators we consider in this paper may have direct applications in various embedding theorems as we see that in one dimension. Analytic function spaces on product domains were under intensive study recently (see, for example, $[7,11,14,15,21,32]$ and various references there). Various other related problems of analytic function theory in tubular domain over symmetric cones were under attention over last two decades and this paper can be also considered as some continuation of that research at the same time.

The theory of one functional analytic spaces on convex and pseudoconvex domains is well-developed by various authors during last decades (see $[8,16,18]$ and various references there). One of the goals of this paper among other things is to define for the first time in literature multifunctional analytic spaces in tubular domains over symmetric cones and to establish some basic properties of these spaces. We believe this new interesting objects can serve as a base for further generalizations and investigations in this active research area. Multifunctional spaces we mentioned above are closely connected also with so-called analytic function spaces on products of strictly tubular domains over symmetric cones $T_{\Omega}^{m}=T_{\Omega} \times \cdots \times T_{\Omega}$. Various such connections in the unit ball in $\mathbb{C}^{n}$ and harmonic function spaces were found and studied recently in $[1,17,26]$. We note basic properties of last spaces on product domains are closely connected on the other hand with so-called Trace operator (see $[1,17])$. Next in the final part of this paper we will turn to the study of certain new embedding theorems for some new mixed norm analytic classes in tubular domains over symmetric cones in $\mathbb{C}^{n}$. At the end of paper various related to this topic remarks will be added.

We denote by $c, c_{1}, c_{\alpha}, \ldots$ various positive constants in various inequalities below.

\section{Basic Preliminaries on Symmetric Cones $\Omega$ and Determinant FUNCTION}

We first shortly remind the readers some basic facts on symmetric cones (see $[2-4,23,24])$. A subset $\Omega$ of $\mathbb{R}^{n}$ or $V$, so that $\operatorname{dim} V=n$ to be a cone if $\lambda x \in \Omega$, for all $x \in \Omega, \lambda>0$, if $\lambda x+\mu y \in \Omega$ for all $x, y \in \Omega, \lambda, \mu>0$ then it is convex. Let in addition $\Omega^{*}=\left\{y \in \mathbb{R}^{n}:(y / x)>0\right.$, for all $\left.x \in \bar{\Omega} \backslash\{0\}\right\}$ and $\Omega^{*}=\Omega$. This type open cone is selfdual ( $\Omega^{*}$ is dual cone).

Let $G(\Omega)=\left\{g \in G l\left(\mathbb{R}^{n}\right): g \Omega=\Omega\right\}$, where $G l\left(\mathbb{R}^{n}\right)$ denotes the group of all linear invertible transformation of $\mathbb{R}^{n}$. If for all $x, y \in \Omega, y=g x$, for some $g \in G(\Omega)$ then our open convex cone $\Omega$ is homogeneous, if also $\Omega^{*}=\Omega$ then it is symmetric cone. These are one of the main objects of this paper.

If the equation $\Omega=\Omega_{1}+\Omega_{2}$ is not possible for each $V_{1} \subset \mathbb{R}^{n}, V_{2} \subset \mathbb{R}^{n}$, then our cone is irreducible, here $V_{i} \neq \varnothing, i=1,2\left(\Omega_{1}, \Omega_{2}\right.$ are symmetric cones), where also $\Omega_{i} \subset V_{i}, i=1,2$. 
We remind shortly the reader now basic facts on determinant $\Delta^{t}(\operatorname{Im} z), z \in \mathbb{C}^{n}$, $t \in(0, \infty)$. We fix $V$ - a simple Euclidean Jordan algebra with rank $r$.

(a) A Jordan algebra $V$ over $R$ is said to be Euclidean if there exists a positive definite bilinear symmetric form on $V$ which is associative $(L(x) u / v)=(u, L(x) v)$, where $(u, v)$ is an inner product on $R^{n}$, for all $x, u, v \in V$;

(b) A Jordan algebra is simple if all it's ideals are trivial;

(c) We define rank of $V$.

If $x \in V, m(x)=\min \left\{k>0:\left(l, x, x^{2}, \ldots, x^{k}\right)\right.$ are linearly dependent $\}$, then $1 \leq m(x) \leq \operatorname{dim} V$ and $r=\max \{m(x): x \in V\}$, we say rank of $V$ is $r$.

According to spectral theorem if $V$ has rank $r$, then $x=\sum_{i=1}^{r} \lambda_{i} c_{i} ; \lambda_{i} \in \mathbb{R} ; c_{i}$ - are elements of so called Jourdan frame, and $\left\{\lambda_{i}\right\}$ are determined uniquely by $x$ (with their multiplicities). We fix now a Peirce decomposition of $V=\oplus_{1 \leq i \leq j \leq r} V_{i j}$; (we formally look at $\mathrm{V}$ as a space of symmetric matrices $\left(V_{i j}\right), V_{i i}=R c_{i}$, where $R$ is a special mapping (see [13]), $V_{i j}=V\left(c_{i}, 1 / 2\right) \cap V\left(c_{i}, 1 / 2\right)=\left\{x \in V: c_{i} x=c_{j} x=\frac{x}{2}\right\}$, $\left.i<j, \operatorname{dim} V_{i j}=d=2 \frac{n / r-1}{r-1}\right)$. We denote by $P_{i j}$ the orthogonal projection of $V$ onto $V_{i j}$ for $i \leq j$. Finally we denote by $\Delta_{j}(x), j=1, \ldots, r$, the principal minors of $x \in V$ with respect to the fixed Jordan frame $\left\{c_{1}, \ldots, c_{r}\right\}$. That is $\Delta_{k}(x)$ is the determinant of the projection $P_{k} x$ of $x$ in the Jordan subalgebra $V^{(k)}=\oplus_{1 \leq i \leq j \leq r} V_{i j}$.

It is well-known that $\Omega=\left\{x \in V: \Delta_{k}(x)>0: k=1, \ldots, r\right\}$. We have also $\Delta_{k}(m x)=\Delta_{k}(x), x \in V, m \in Z_{+}, m>0$. See other properties of $\Delta_{k}$ in [2,24].

We define $\Delta_{s}(x)=\prod_{j=1}^{r} \Delta_{j}^{s_{j}-s_{j}+1}(x)=\Delta_{1}^{s_{1}-s_{2}}(x) \ldots \Delta_{r}^{S_{r}}(x), x \in \Omega, s \in C^{r}$. We have that $\left|\Delta_{s}\right|=\Delta(\operatorname{Im} z)$ and $\Delta_{s} \sum_{i=1}^{r} a_{i} c_{i}=\prod_{i=1}^{r} a_{i}^{s_{i}} ; a_{i}>0, i=1, \ldots, r$.

To formulate our theorems we will need basic facts of theory of analytic function spaces in tubular domains over symmetric cones taken from $[4,23,24]$. Let $d v(w)$ and $d v_{\alpha}(w)=\left[\Delta^{\alpha-\frac{n}{r}}(v)\right] d u d v, \alpha>n / r-1$, be a standard Lebesque measure in tubular domains over symmetric cone $T_{\Omega}$ and weighted Lebesque measure in tube, $w=u+i v$. The weighted Bergman kernel $B_{\nu}$ of $T_{\Omega}$ is given as usual by

$$
B_{\nu}(w, z)=\left(d_{\nu}\right) \Delta\left(\frac{w-\bar{z}}{i}\right)^{-\nu-\frac{n}{r}}, \quad w, z \in T_{\Omega}, \quad \nu \in \mathbb{R},
$$

is a Bergman constant, where

$$
d_{\nu}=\left(c_{\nu}^{-1}\right) \Gamma\left(\nu+\frac{n}{r}\right) .
$$

Let $\Omega$ be an irreducible symmetric cone in the Euclidean space $V$, and $T_{\Omega}=V+i \Omega$ the corresponding tube domain in the complexified space $V^{\mathbb{C}}$. We shall note $n$ the dimension of $V$ and $r$ the rank of $\Omega$. Moreover, we shall denote by $(x \mid y)$ the scalar product in $V$, and by $\Delta$ the determinant function. For the description of such cones $\Omega$ in terms of Jordan algebras, one may use the book of Faraut and Koranyi [13]. One may also have in mind the typical example that one obtains when $V$ is the space of real symmetric $r \times r$ matrices, and $\Omega$ is the cone of positive definitive matrices. In this example, the scalar product on $V$ is induced by the Hilbert-Schmidt norm of the 
matrices, and the determinant function is given by the determinant of the matrices. The rank is $r$, while the dimension is $\frac{r(r+1)}{2}$.

We shall also make use the generalized wave operator on $V$, given by $\square=\Delta\left(\frac{1}{i} \frac{\partial}{\partial x}\right)$. This is a differential operator of degree $r$, defined by the equality

$$
\Delta\left(\frac{1}{i} \frac{\partial}{\partial x}\right)\left[e^{i(z \mid \zeta)}\right]=\Delta(\zeta) e^{i(x \mid \zeta)}, \quad \zeta \in V
$$

It's name is due to another fundamental example, given by the forward light cone in $\mathbb{R}^{n}$,

$$
\left\{x \in \mathbb{R}^{n} ; x_{1}>\sqrt{x_{2}^{2}+\cdots+x_{n}^{2}}\right\}
$$

which is of rank 2. In this case, the determinant function is equal to

$$
\Delta(x)=x_{1}^{2}-x_{2}^{2}-\cdots-x_{n}^{2}
$$

\section{Basic Lemmas and Theorems on Bergman Spaces and Bergman Type INTEGRAL OPERATORS}

In this section several known assertions on determinant function, Bergman kernel, Bergman spaces will be given. Almost all assertions will be used by us later.

Let $T_{\Omega}$ be the tube domain over symmetric cone, and $H\left(T_{\Omega}\right)$ be the space of all analytic functions in tube (see [2]). We define Bergman spaces for $1 \leq p, q<\infty, \gamma\rangle$ $\frac{n}{r}-1$. Let

$$
\left(A_{\gamma}^{p, q}\right)\left(T_{\Omega}\right)=\left\{f \in H\left(T_{\Omega}\right):\left(\int_{\Omega}\left(\int_{\mathbb{R}^{n}}|f(x+i y)|^{p} d x\right)^{\frac{q}{p}}\left(\Delta^{\gamma-\frac{n}{r}}(y)\right) d y\right)^{\frac{1}{q}}<\infty\right\} .
$$

Replacing $A$ by $L$ and $H$ by $L^{1}$ we get as usual known larger spaces of measurable functions in tube $T_{\Omega}$. Note $\left(A_{\gamma}^{p, q}\right)=\{0\}$ if $\gamma \leq \frac{n}{r}-1$ (see [2]).

These spaces are Banach spaces. We will provide below some known facts in these analytic spaces in tube $T_{\Omega}$. We will need them for our proofs below.

Lemma 3.1 (Whitney decomposition of the cone $\Omega$, see [2]). Let $\delta>0$. Given $y \in \Omega$ we denote $B_{\delta}(y)=\{\zeta \in \Omega: d(\zeta, y)<\delta\}$; where $d:(\Omega \times \Omega) \rightarrow\left(R_{+}\right)$is a distance function, see [2]. There exist a sequence of points of $\Omega$ such that the following properties hold.

1) The balls $B_{\delta}\left(\zeta_{k}\right)$ are pairwise disjoint;

2) the balls $B_{\delta}\left(\zeta_{k}\right)$ form a covering of $\Omega$;

3) there is an integer $N=N(\Omega)$ such that every $y \in \Omega$ belongs to at most $N$ balls $B_{\delta}\left(\zeta_{k}\right)$ (finite overlapping property).

As a consequence we have [2] a natural covering of the cone and $R_{+}$with invariant balls and the dyadic decomposition of $(0, \infty),\left(2^{j-1}, 2^{j+1}\right)=\left[B_{\log 2}\left(2^{j}\right)\right]=\{p \in \Omega$ : $\left.d\left(p, 2^{j}\right)<\log 2\right\}, j \in Z$ (see [2]).

Let $B_{\delta}\left(z_{j}\right)=B_{j}$ be the Bergman ball in tube (see [2]). 
Lemma 3.2 (Whitney decomposition of tube, see [2]). Given $\delta \in(0,1]$ there exist a sequence $\left\{z_{j}\right\}$ of points of $T_{\Omega}$ such that if $B_{j}=B_{\delta}\left(z_{j}\right): B_{j}^{\prime}=B_{\delta / 3}\left(z_{j}\right)$

1) the balls $\left(B_{j}^{\prime}\right)$ are pairwise disjoint;

2) the balls $\left(B_{j}\right)$ form a cover of $T_{\Omega}$;

3) there exists a positive integer $N=N(\Omega)$ (independent of $\delta$ ) such that every point of $T_{\Omega}$ belongs to at most $N$ balls $B_{j}$.

We provide now a vital application of the first lemma.

Lemma 3.3 (see [2]). Let $\gamma>\frac{n}{r}-1 ; 1 \leq p, q<\infty$. Then for every lattice $\left\{y_{i}\right\}$ in $\Omega$ there exist constant $c>0$ such that

$$
\frac{1}{c}\left(\|f\|_{A_{\gamma}^{p, q}}\right) \leq\left(\sum_{j}\left(\Delta^{\gamma}\left(y_{j}\right)\right)\left\|f\left(x+i y_{j}\right)\right\|_{p}^{q}\right)^{\frac{1}{q}} \leq c\left(\|f\|_{A_{\gamma}^{p, q}}\right), \quad \text { for all } f \in A_{\gamma}^{p, q}\left(T_{\Omega}\right),
$$

where the norm in the middle is the Hardy space norm.

The sequence of points $\left\{z_{i}\right\}$ is called a $\delta$ lattice of $T_{\Omega}$, we have also (see [2])

$$
v_{\alpha}\left(B_{j}\right) \asymp v_{\alpha}\left(B_{j}^{\prime}\right) \asymp \Delta^{\left(\frac{2 n}{r}\right)+\alpha}\left(\operatorname{Im} z_{j}\right), \quad \alpha>\frac{n}{r}-1, \quad z_{j} \in T_{\Omega}, \quad j=1, \ldots, n .
$$

Lemma 3.4 (Estimates for $\Delta^{t}$ function and Bergman kernel, see[2]).

1) Let $\lambda>\frac{n}{r}-1$ be fixed. Then $\Delta\left(y+y^{\prime}\right) \geq \Delta(y)$; for all $y, y^{\prime} \in \Omega,\left|\Delta^{-\lambda}\left(\frac{x+i y}{i}\right)\right| \leq$ $\left(\Delta(y)^{-\lambda}\right) ;$ for all $x \in \mathbb{R}^{n} ; y \in \Omega$.

2) Let $\alpha, \beta$ real, then

$$
I_{\alpha, \beta}(t)=\int_{\Omega}\left(\Delta^{\alpha}(y+t)\right)\left(\Delta^{\beta}(y)\right) d y<\infty
$$

if $\beta>-1, \alpha+\beta<\left(-\frac{2 n}{r}+1\right)$, and

$$
I_{\alpha, \beta}(t)=\left(c_{\alpha, \beta}\right) \Delta^{\alpha+\beta+\frac{n}{r}}(t) .
$$

Moreover

$$
I_{\alpha}(y)=\int_{\mathbb{R}^{n}}\left|\Delta^{-\alpha}\left(\frac{x+i y}{i}\right)\right| d x<\infty
$$

if $\alpha>\frac{2 n}{r}-1$; and

$$
I_{\alpha}(y)=c_{\alpha}\left(\Delta^{-\alpha+\frac{n}{r}}(y)\right)
$$

where $y \in \Omega$.

3) For $\beta>-1, \alpha>\frac{n}{r}-1, z=x+i y, w=u+i v, w, z \in T_{\Omega}$ we have

$$
\int_{T_{\Omega}}\left(\Delta^{\beta}(y)\right)\left|B_{\alpha+\beta+\frac{n}{r}}(z, w)\right| d v(z) \leq c \Delta^{-\alpha}(v), \quad v \in \Omega
$$


Lemma 3.5 (Reproducing formulas, see [2]). For any analytic function from $A_{\alpha}^{2}\left(T_{\Omega}\right)$ the following integral formula is valid

$$
f(z)=\widetilde{c}_{\alpha} \int_{T_{\Omega}} B_{\alpha}(z, w)(f(w)) d v_{\alpha}(w), \quad z \in T_{\Omega} .
$$

Let $1 \leq p<\infty, 1 \leq q<\infty ;(n / r) \leq p_{1} ; \frac{1}{p_{1}}+\frac{1}{p}=1 ;\left(\frac{n}{r}-1\right)<\gamma$.

Let $f \in A_{\gamma}^{p, q}$ then (3.3) with $\alpha>\left(\frac{n}{r}-1\right)$ is valid (Bergman representation formula with $\alpha$ index is valid).

We provide below a well-known and important application of r-lattices of tubular domains over symmentic cones.

Lemma 3.6 (Atomic decomposition of $A_{\gamma}^{p}$, see [2]). Let $p>=1$, and $\nu>\frac{n}{r}-1$. Let $\left\{z_{j}\right\}$ be a $\delta$-lattice in $T_{\Omega} ; \delta \in(0,1) ; z_{j}=x_{j}+i y_{j}, z_{j} \in T_{\Omega}, j=1, \ldots, r$. Then

$$
\|f\|_{A_{\nu}^{p}} \asymp \sum_{j}\left|f\left(z_{i}\right)\right|^{p} \Delta^{\nu+\frac{n}{r}}\left(y_{i}\right) .
$$

Assume that Bergman projection $P_{\nu}$ is bounded on $A_{\nu}^{p}$ and let $\left\{z_{j}\right\}$ be a $\delta$-lattice in $T_{\Omega}$. Then if $f \in A_{\nu}^{p}$ then

$$
\begin{aligned}
& f(z)=\sum_{j} \lambda_{j} B_{\nu}\left(z, z_{j}\right) \Delta^{\nu+\frac{n}{r}}\left(y_{i}\right), z \in T_{\Omega} \\
& \left.\sum_{j=1}^{\infty}\left|\lambda_{j}\right|^{p} \Delta^{\nu+\frac{n}{r}}\left(y_{j}\right)\right) \leq c\|f\|_{A_{\nu}^{p}}^{p} .
\end{aligned}
$$

If

$$
\sum_{j=1}^{\infty}\left|\lambda_{j}\right|^{p}\left(\Delta^{\nu+\frac{n}{r}}\left(y_{j}\right)\right)<\infty,
$$

then the "sum with $B_{\nu}$ " (3.4) converges in $A_{\nu}^{p}$ and the reverse to (3.5) is true also.

We mention now several known results on Bergman type projections . The weighted Bergman projection $P_{\nu}$ is the orthogonal projection from the Hilbert space $L_{\nu}^{2}\left(T_{\Omega}\right)$ onto its closed subspace $A_{\nu}^{2}\left(T_{\Omega}\right)$ and it is given by the integral formula

$$
\left(P_{\nu} f\right)(z)=\int_{T_{\Omega}} B_{\nu}(z, w) f(w) \Delta^{\nu-\frac{n}{r}}(\operatorname{Im} w) d v(w),
$$

$z \in T_{\Omega}, \nu>\frac{n}{r}-1$ (see $\left.[2-4,23,24]\right)$.

The $L_{\nu}^{p, q}$ boundedness of the Bergman projection $P_{\nu}$ is still an open problem and has attracted a lot of attention in recent years. Today it is only known that this projection extends to a bounded operator on $L_{\nu}^{p, q}$ for general symmetric cones for the range $1 \leq p<\infty, q_{\nu, p}^{\prime}<q<q_{\nu, p} ; q_{\nu, p}=\min \left\{p, p^{\prime}\right\} q_{\nu}, q_{\nu}=1+\frac{\nu}{n / r-1}$ and $\frac{1}{p}+\frac{1}{p^{\prime}}=1$ (see $[23,24])$. 
The importance can be seen for example from the following fact. If $P_{\nu}$ extends to a bounded operator on $L_{\nu}^{p, q}$ then the topological dual space $\left(A_{\nu}^{p, q}\right)^{*}$ of the Bergman space $A_{\nu}^{p, q}$ identifies with $A_{\nu}^{p^{\prime}, q^{\prime}}$ under the integral pairing

$$
\langle f, g\rangle_{\nu}=\int_{T_{\Omega}} f(z) \overline{g(z)} \Delta^{\nu-\frac{n}{r}}(\operatorname{Im} z) d v(z)
$$

$f \in A_{\nu}^{p, q} ; g \in A_{\nu}^{p^{\prime}, q^{\prime}}($ see $[23,24])$. Let

$$
\begin{aligned}
& \left(T_{\alpha, \beta, \gamma} f\right)(z)=\Delta^{\alpha}(\operatorname{Im} z) \int_{T_{\Omega}} B_{\gamma}(z, w) f(w) \Delta^{\beta}(\operatorname{Im} w) d v(w), \\
& \left(T_{\alpha, \beta, \gamma}^{+} f\right)(z)=\Delta^{\alpha}(\operatorname{Im} z) \int_{T_{\Omega}}\left|B_{\gamma}(z, w)\right| f(w) \Delta^{\beta}(\operatorname{Im} w) d v(w),
\end{aligned}
$$

$z \in T_{\Omega}, f \in L^{1}(T \Omega)$. The following assertions were proved in [24].

Theorem 3.1. There are $\nu_{1}=\nu_{1}(\alpha, n, r, q) ; \nu_{2}=\nu_{2}(\alpha, n, r, q)$ so that for $1 \leq p, q<$ $\infty, \nu \in \mathbb{R}, \gamma=\alpha+\beta+\frac{n}{r}, \alpha+\beta>-1$ then $T_{\alpha, \beta, \gamma}^{+}$is a bounded operator on $L_{\nu}^{p, q}\left(T_{\Omega}\right)$ for all $\nu \in\left(\nu_{1}, \nu_{2}\right)$.

Theorem 3.2. Let $\left(Q^{+}\right)$be $\left(T_{\alpha, \beta, \gamma}^{+}\right)$operator for $\alpha=0, \gamma=\nu+m ; \beta=\nu-\frac{n}{r}$. Then $\left(Q^{+}\right)$for $\nu+m>\frac{n}{r}-1,1 \leq p, q<\infty$, is a bounded operator from $L_{\nu}^{p, q}$ to $L_{\nu+m q}^{p, q}$ if $\nu \in\left(\nu_{1}, \nu_{2}\right)$ for some $\nu_{1}=\nu_{1}(p, q, n, r, \nu) ; \nu_{2}=\nu_{2}(p, q, n, r, \nu),\left(T_{\alpha, \beta, \gamma}^{+}\right)$is a bounded operator on $L^{\infty}$ if $\alpha>\frac{n}{r}-1, \beta>-1, \gamma=\alpha+\beta+\frac{n}{r}$. The same is valid for $T_{\alpha, \beta, \gamma}$ operator.

To formulate our theorems in next section we will consider various similar Bergman type operators on products and semiproducts of tubular domains over symmetric cones. Note our proofs are much simpler than parallel proofs in [24]. We, in particular, will consider the following integral operators in functional classes in tube.

Let further

$$
\left(T_{\beta} h\right)(\vec{w})=\underbrace{\int_{\mathbb{R}^{n}} \cdots \int_{\mathbb{R}^{n}}}_{m} \int_{\Omega} \frac{h\left(x_{1}+i y, \ldots, x_{m}+i y\right) \Delta^{\beta}(y)}{\prod_{j=1}^{m} \Delta^{\frac{\beta+n / r+m n / r}{m}}\left(\frac{\bar{x}_{j}+i \bar{y}-w_{j}}{i}\right)} d y d x_{1} \ldots d x_{m},
$$

where $\vec{w}=\left\{w_{1}, \ldots, w_{m}\right\}=\left\{\zeta_{1}+i \eta, \ldots, \zeta_{m}+i \eta\right\} \in T_{\Omega}$

$$
\left(\tilde{T}_{\vec{\beta}} h\right)(\vec{w})=\int_{\Omega^{m}} \int_{\mathbb{R}^{n}} \frac{h\left(x+i y_{1}, \ldots, x+i y_{m}\right) \Delta^{\beta_{1}}\left(y_{1}\right) \ldots \Delta^{\beta_{m}}\left(y_{m}\right)}{\prod_{j=1}^{m} \Delta^{\beta_{j}+\frac{n / r+m n / r}{m}}\left(\frac{\bar{x}+i \bar{y}_{j}-w_{j}}{i}\right)} d x d y_{1} \ldots d y_{m}
$$

where $\vec{w}=\left\{w_{1}, \ldots, w_{m}\right\}=\left\{\zeta+i \eta_{1}, \ldots, \zeta+i \eta_{m}\right\} \in T_{\Omega}$, and $\vec{\beta}=\left\{\beta_{1}, \ldots, \beta_{m}\right\}$, where $\beta>\frac{n}{r}-1, \beta_{j}>\frac{n}{r}-1, j=1, \ldots, m, h \in L^{1}\left(T_{\Omega}^{m}\right)$. Complete analogues of these operators were introduced and studied in [27] in the unit disk. These operators, in particular, can be considered as direct extensions of classical and well-studied Bergman projection in tubular domains over symmetric cones. 


\section{New Integral Operators in Bergman-type Analytic Spaces on Semi-Products of Tubular Domains Over Symmetric Cones}

This section is devoted to formulations and proofs of all our main results related to integral operators. We, in particular, extend some results provided by us in previous section. Note our results of this type may have various applications in function theory and also in operator theory.

The following theorem gives a new complete extension of Theorem 1.1 to semiproduct of tubular domain over symmetric cones.

Note the complete analogue of Theorem 4.1 in the unit disk can be found in [27].

Theorem 4.1. Let $1<p<\infty, \beta>m \frac{m}{r}-m-\frac{n}{r}+\frac{m n p}{r(p-1)}, \tau>(p-1)\left(\frac{2 m n}{r}-m-\frac{n}{r}\right)-1$ and $m \in \mathbb{N}, m>1, \tau-p \beta<1-\frac{n}{r}$ then

$$
\begin{aligned}
& \underbrace{\int_{\mathbb{R}^{n}} \ldots \int_{\mathbb{R}^{n}}}_{m} \int_{\Omega}\left|\left(T_{\beta} h\right)(\vec{w})\right|^{p} \Delta^{\tau}(\eta) d \eta d \zeta_{1} \ldots d \zeta_{m} \\
\leq & c \underbrace{\int_{\mathbb{R}^{n}} \cdots \int_{\mathbb{R}^{n}}}_{m} \int_{\Omega}|h(\vec{z})|^{p} \Delta^{\tau}(y) d y d x_{1} \ldots d x_{m},
\end{aligned}
$$

where $\vec{w}=\left\{w_{1}, \ldots, w_{m}\right\}=\left\{\zeta_{1}+i \eta, \ldots, \zeta_{m}+i \eta\right\} \in T_{\Omega}^{m}, \vec{z}=\left\{z_{1}, \ldots, z_{m}\right\}=\left\{x_{1}+\right.$ $\left.i y, \ldots, x_{m}+i y\right\} \in T_{\Omega}^{m}$.

Proof. We have

$$
\begin{aligned}
I(h)= & \underbrace{\int_{\mathbb{R}^{n}} \cdots \int_{\mathbb{R}^{n}}}_{m} \int_{\Omega}\left|\left(T_{\beta} h\right)(\vec{w})\right|^{p} \Delta^{\tau}(\eta) d \eta d \zeta_{1} \ldots d \zeta_{m} \\
= & \underbrace{\int_{\mathbb{R}^{n}} \cdots \int_{\mathbb{R}^{n}}}_{m} \int_{\Omega}|\underbrace{\int_{\mathbb{R}^{n}} \ldots \int_{\mathbb{R}^{n}}}_{m} \int_{\Omega} \frac{h\left(x_{1}+i y, \ldots, x_{m}+i y\right) \Delta^{\beta}(y)}{\prod_{j=1}^{m} \Delta^{\frac{\beta+n / r+m n / r}{m}}\left(\frac{\bar{x}_{j}+i \bar{y}-w_{j}}{i}\right)} d y d x_{1} \ldots d x_{m}|^{p} \\
& \times \Delta^{\tau}(\eta) d \eta d \zeta_{1} \ldots d \zeta_{m} .
\end{aligned}
$$

Denote

$$
M(h)=|\underbrace{\int_{\mathbb{R}^{n}} \ldots \int_{\mathbb{R}^{n}}}_{m} \int_{\Omega} \frac{h\left(x_{1}+i y, \ldots, x_{m}+i y\right) \Delta^{\beta}(y)}{\prod_{j=1}^{m} \Delta^{\frac{\beta+n / r+m n / r}{m}}\left(\frac{\bar{x}_{j}+i \bar{y}-w_{j}}{i}\right)} d y d x_{1} \ldots d x_{m}|^{p} .
$$


We add and subtract $2+\varepsilon$ below

$$
M(h)=|\underbrace{\int_{\mathbb{R}^{n}} \ldots \int_{\mathbb{R}^{n}}}_{m} \int_{\Omega} \frac{h\left(x_{1}+i y, \ldots, x_{m}+i y\right) \Delta^{\beta}(y)}{\prod_{j=1}^{m} \Delta^{\frac{\beta+n / r+m n / r}{m}+2+\varepsilon-2-\varepsilon}\left(\frac{\bar{x}_{j}+i \bar{y}-w_{j}}{i}\right)} d y d x_{1} \ldots d x_{m}|^{p} .
$$

By Holder's inequality with power $p$ we have $\left(\frac{1}{p}+\frac{1}{p^{\prime}}=1\right)$,

$$
\begin{aligned}
& M(h)=|\underbrace{\int_{\mathbb{R}^{n}} \ldots \int_{\mathbb{R}^{n}}}_{m} \int_{\Omega} \frac{h\left(x_{1}+i y, \ldots, x_{m}+i y\right) \Delta^{\beta}(y)}{\prod_{j=1}^{m} \Delta^{\frac{\beta+n / r+m n / r}{m}+\frac{2}{p}+\frac{2}{p^{\prime}}+\varepsilon-2-\varepsilon}\left(\frac{\bar{x}_{j}+i \bar{y}-w_{j}}{i}\right)} d y d x_{1} \ldots d x_{m}|^{p} \\
& \leq(\underbrace{\int_{\mathbb{R}^{n}} \cdots \int_{\mathbb{R}^{n}}}_{m} \int_{\Omega} \frac{\left|h\left(x_{1}+i y, \ldots, x_{m}+i y\right)\right|^{p} \Delta^{p \beta}(y)}{\prod_{j=1}^{m}\left|\Delta\left(\frac{\beta+n / r+m n / r}{m}-2-\varepsilon\right) p+2\left(\frac{\bar{x}_{j}+i \bar{y}-w_{j}}{i}\right)\right|} d y d x_{1} \ldots d x_{m}) \\
& \times(\underbrace{\int_{\mathbb{R}^{n}} \cdots \int_{\mathbb{R}^{n}}}_{m} \int_{\Omega} \frac{d y d x_{1} \ldots d x_{m}}{\prod_{j=1}^{m}\left|\Delta^{\varepsilon p^{\prime}+2}\left(\frac{\bar{x}_{j}+i \bar{y}-w_{j}}{i}\right)\right|})^{p / p^{\prime}} .
\end{aligned}
$$

By (3.1) and (3.2) the last factor can be calculated as follows

$$
(\underbrace{\int_{\mathbb{R}^{n}} \cdots \int_{\mathbb{R}^{n}}}_{m} \int_{\Omega} \frac{d y d x_{1} \ldots d x_{m}}{\prod_{j=1}^{m}\left|\Delta^{\varepsilon p^{\prime}+2}\left(\frac{\bar{x}_{j}+i \bar{y}-w_{j}}{i}\right)\right|})^{p / p^{\prime}}=c \Delta^{\left(-\varepsilon p^{\prime} m-2 m+m n / r+n / r\right) p / p^{\prime}}(\eta),
$$

if $\varepsilon p^{\prime}+2>\frac{2 n}{r}-1$ and $m\left(\varepsilon p^{\prime}+2\right)-\frac{m n}{r}>\frac{2 n}{r}-1$. We have to choose now a correct lower bound for $\varepsilon$ in order to satisfy both our estimates which we need to use above. An easy technical calculation shows that the following bound will satisfy our purposes

$$
\varepsilon>\frac{3 n}{p^{\prime} r}-\frac{3}{p^{\prime}} .
$$

Finally we have

$$
\begin{aligned}
I(h) & \leq \underbrace{c}_{m} \ldots \int_{\mathbb{R}^{n}} \int_{\Omega} \Delta^{\left(-\varepsilon p^{\prime} m-2 m+m n / r+n / r\right) p / p^{\prime}+p \beta}(\eta) \\
& \times \underbrace{\int_{\mathbb{R}^{n}} \cdots \int_{\mathbb{R}^{n}}}_{m} \int_{\Omega} \frac{\left|h\left(x_{1}+i y, \ldots, x_{m}+i y\right)\right|^{p} \Delta^{p \beta}(y)}{\prod_{j=1}^{m}\left|\Delta^{\left(\frac{\beta+n / r+m n / r}{m}-2-\varepsilon\right) p+2}\left(\frac{\bar{x}_{j}+i \bar{y}-w_{j}}{i}\right)\right|} d y d x_{1} \ldots d x_{m} d \eta d \zeta_{1} \ldots d \zeta_{m}
\end{aligned}
$$


Applying Fubbini's theorem we have

$$
\begin{aligned}
I(h) & \leq c \underbrace{\int_{\mathbb{R}^{n}} \ldots \int_{\mathbb{R}^{n}}}_{m} \int_{\Omega}\left|h\left(x_{1}+i y, \ldots, x_{m}+i y\right)\right|^{p} \Delta^{p \beta}(y) \\
& \times \underbrace{\int_{\mathbb{R}^{n}} \cdots \int_{\mathbb{R}^{n}}}_{m} \int_{\Omega} \frac{\Delta^{\left(-\varepsilon p^{\prime} m-2 m+m n / r+n / r\right) p / p^{\prime}+\tau}(\eta)}{\prod_{j=1}^{m}\left|\Delta^{\left(\frac{\beta+n / r+m n / r}{m}-2-\varepsilon\right) p+2}\left(\frac{\bar{x}_{j}+i \bar{y}-w_{j}}{i}\right)\right|} d \eta d \zeta_{1} \ldots d \zeta_{m} d y d x_{1} \ldots d x_{m} .
\end{aligned}
$$

Note that we can apply (3.1) and (3.2) again for the following values of parameters.

$$
\begin{gathered}
\frac{p \beta}{m}+\frac{n p}{r m}+\frac{n p}{r}-2 p-\varepsilon p+2>\frac{2 n}{r}-1, \\
-\varepsilon p m-\frac{2 m p}{p^{\prime}}+\frac{m n p}{r p^{\prime}}+\frac{n p}{r p^{\prime}}+\tau>-1, \\
\tau-\varepsilon p m-2 m p / p^{\prime}+m n p /\left(r p^{\prime}\right)+n p /\left(r p^{\prime}\right)-\beta p-p n / r-m p n / r
\end{gathered}
$$

$$
+2 m p+\varepsilon m p-2 m+m n / r<-\frac{2 n}{r}+1 .
$$

Looking at (4.2) with respect to (4.1) we have

$$
\begin{aligned}
& \frac{p \beta}{m}+\frac{n p}{m r}+\frac{n p}{r}-2 p-\frac{3 n p}{p^{\prime} r}+3 \frac{p}{p^{\prime}}+2>\frac{2 n}{r}-1, \\
& \frac{p p^{\prime} \beta r+n p p^{\prime}+n m p p^{\prime}-2 p m r p^{\prime}-3 n p m+3 p m r+2 m r p^{\prime}-2 n m p^{\prime}+m r p^{\prime}}{m r p^{\prime}}>0, \\
& \beta r+n-n m+m r-\frac{n p}{p^{\prime}}>0, \\
& \beta>m \frac{n}{r}-m-\frac{n}{r}+\frac{m n p}{r(p-1)} .
\end{aligned}
$$

Looking at (4.3) with respect to (4.1) we have

$$
\tau>(p-1)\left(\frac{2 m n}{r}-m-\frac{n}{r}\right)-1
$$

We will heavily simplify estimate (4.4) below.

We have

$$
\begin{aligned}
& \underbrace{\int_{\mathbb{R}^{n}} \cdots \int_{\mathbb{R}^{n}}}_{m} \int_{\Omega} \frac{\Delta^{\left(-\varepsilon p^{\prime} m-2 m+m n / r+n / r\right) p / p^{\prime}+\tau}(\eta)}{\prod_{j=1}^{m}\left|\Delta^{\left(\frac{\beta+n / r+m n / r}{m}-2-\varepsilon\right) p+2}\left(\frac{\bar{x}_{j}+i \bar{y}-w_{j}}{i}\right)\right|} d \eta d \zeta_{1} \ldots d \zeta_{m} \\
= & c_{1}(\Delta y)^{p \beta-\varepsilon p m-2 m p / p^{\prime}+m n p /\left(r p^{\prime}\right)+n p /\left(r p^{\prime}\right)-\beta p r / n-p-m p+2 m p+\varepsilon m p-2 m+m n / r+n / r} .
\end{aligned}
$$

But given $p p^{\prime}=p+p^{\prime}$ we obtain

$$
\begin{aligned}
\tau-\varepsilon p m-2 m p / p^{\prime}+m n p /\left(r p^{\prime}\right)+n p /\left(r p^{\prime}\right) & -\beta p-p n / r-m p n / r+2 m p \\
& +\varepsilon m p-2 m+m n / r+n / r=\tau-p \beta .
\end{aligned}
$$


Now looking simply at the previous page and combining the estimates from there we arrive at the new estimate

$$
\tau-p \beta-\frac{n}{r}<-\frac{2 n}{r}+1 ; \quad \tau-p \beta<-\frac{n}{r}+1 .
$$

This means

$$
I(h) \leq c_{2} \underbrace{\int_{\mathbb{R}^{n}} \cdots \int_{\mathbb{R}^{n}}}_{m} \int_{\Omega}\left|h\left(x_{1}+i y, \ldots, x_{m}+i y\right)\right|^{p} \Delta^{p \beta+\tau-p \beta}(y) d y d x_{1} \ldots d x_{m} .
$$

This completes the proof of our assertion.

We can prove almost similarly the following theorem for related $\tilde{T}_{\beta}$ operator. We omit the technical proof leaving it to interested readers. Note complete analogue of Theorem 4.2 in case of unit disk can be seen in [27].

Theorem 4.2. Let $\beta_{j}>\frac{n}{r}-1, j=1, \ldots, m, 1<p<\infty, \beta_{j}>2+\frac{n}{r}-\frac{2 n}{p r}-\frac{3}{m}-\frac{2}{p}+$ $\frac{n}{r m p}+\frac{2}{m p}, \tau_{j}>\frac{n}{r} p-p-\frac{n}{r}, \tau_{j}-p \beta_{j}<1-\frac{n}{r}, j=1, \ldots, m$, then

$$
\begin{aligned}
& \int_{\Omega^{m}} \int_{\mathbb{R}^{n}}\left|\left(\tilde{T}_{\vec{\beta}} h\right)(\vec{w})\right|^{p} \Delta^{\tau_{1}}\left(\eta_{1}\right) \times \cdots \times \Delta^{\tau_{m}}\left(\eta_{m}\right) d \zeta d \eta_{1} \ldots d \eta_{m} \\
\leq & c \int_{\Omega^{m}} \int_{\mathbb{R}^{n}}|h(\vec{z})|^{p} \Delta^{\tau_{1}}\left(y_{1}\right) \times \cdots \times \Delta^{\tau_{m}}\left(y_{m}\right) d x d y_{1} \ldots d y_{m},
\end{aligned}
$$

where $\vec{w}=\left\{\zeta+i \eta_{1}, \ldots, \zeta+i \eta_{m}\right\}, \vec{z}=\left\{z_{1}, \ldots, z_{m}\right\}=\left\{x+i y_{j}, \ldots, z_{m}+i y_{j}\right\}, \vec{z} \in T_{\Omega}^{m}$, $\vec{w} \in T_{\Omega}^{m}$.

Proof. Here is a short scheme of the proof. Denote

$I(h)=\int_{\Omega^{m}} \int_{\mathbb{R}^{n}}\left|\left(\tilde{T}_{\vec{\beta}} h\right)(\vec{w})\right|^{p} \Delta^{\tau_{1}}\left(\eta_{1}\right) \times \cdots \times \Delta^{\tau_{m}}\left(\eta_{m}\right) d \zeta d \eta_{1} \ldots d \eta_{m}, \quad M=\left|\left(\tilde{T}_{\vec{\beta}} h\right)(\vec{w})\right|^{p}$.

Let add and subtract $2+\varepsilon$ in denominator's power:

$$
M(h)=\left|\int_{\Omega^{m}} \int_{\mathbb{R}^{n}} \frac{h\left(x+i y_{j}, \ldots, x+i y_{j}\right) \Delta^{\beta_{1}}\left(y_{1}\right) \ldots \Delta^{\beta_{m}}\left(y_{m}\right)}{\prod_{j=1}^{m} \Delta^{\beta_{j}+\frac{n / r+m n / r}{m}+2+\varepsilon-2-\varepsilon}\left(\frac{\bar{x}+i \bar{y}_{j}-w_{j}}{i}\right)} d x d y_{1} \ldots d y_{m}\right|^{p} .
$$

By Holders' inequality with power $p$ we have $\left(\frac{1}{p}+\frac{1}{p^{\prime}}=1\right)$

$$
\begin{aligned}
& M(h) \\
\leq & \left(\int_{\Omega^{m}} \int_{\mathbb{R}^{n}} \frac{\left|h\left(x+i y_{j}, \ldots, x+i y_{j}\right)\right|^{p} \Delta^{p \beta_{1}}\left(y_{1}\right) \times \cdots \times \Delta^{p \beta_{m}}\left(y_{m}\right)}{\prod_{j=1}^{m}\left|\Delta^{\left(\beta_{j}+\frac{n / r+m n / r}{m}-2-\varepsilon\right) p+2}\left(\frac{\bar{x}+i \bar{y}_{j}-w_{j}}{i}\right)\right|} d x d y_{1} \ldots d y_{m}\right)
\end{aligned}
$$




$$
\begin{aligned}
& \times\left(\int_{\Omega^{m}} \int_{\mathbb{R}^{n}} \frac{d x d y_{1} \ldots d y_{m}}{\prod_{j=1}^{m}\left|\Delta^{\varepsilon p^{\prime}+2}\left(\frac{\bar{x}+i \bar{y}_{j}-w_{j}}{i}\right)\right|}\right)^{p / p^{\prime}} \\
\leq & \left(\int_{\Omega^{m}} \prod_{j=1}^{m}\left(\int_{\mathbb{R}^{n}} \frac{d x}{\left|\Delta^{m \varepsilon p^{\prime}+2 m}\left(\frac{\bar{x}+i \bar{y}_{j}-w_{j}}{i}\right)\right|}\right)^{\frac{1}{m}} d y_{1} \ldots d y_{m}\right)^{p / p^{\prime}} .
\end{aligned}
$$

By (3.1) and (3.2) with restriction for $\varepsilon$ we obtain

$$
\begin{aligned}
& \left(\int_{\Omega^{m}} \prod_{j=1}^{m}\left(\int_{\mathbb{R}^{n}} \frac{d x}{\left|\Delta^{m \varepsilon p^{\prime}+2 m}\left(\frac{\bar{x}+i \bar{y}_{j}-w_{j}}{i}\right)\right|}\right)^{\frac{1}{m}} d y_{1} \ldots d y_{m}\right)^{p / p^{\prime}} \\
= & c \Delta^{\left(-\varepsilon p^{\prime}-2+n /(r m)+n / r\right) p / p^{\prime}}\left(\eta_{1}\right) \times \cdots \times \Delta^{\left(-\varepsilon p^{\prime}-2+n /(r m)+n / r\right) p / p^{\prime}}\left(\eta_{m}\right) .
\end{aligned}
$$

So

$$
\begin{aligned}
I(h) \leq & c_{1} \int_{\Omega^{m}} \int_{\mathbb{R}^{n}} \Delta^{\left(-\varepsilon p^{\prime}-2+n /(r m)+n / r\right) p / p^{\prime}+\tau_{1}}\left(\eta_{1}\right) \\
& \times \Delta^{\left(-\varepsilon p^{\prime}-2+n /(r m)+n / r\right) p / p^{\prime}+\tau_{m}}\left(\eta_{m}\right) \\
\times & \int_{\Omega^{m}} \int_{\mathbb{R}^{n}} \frac{\left|h\left(x+i y_{j}, \ldots, x+i y_{j}\right)\right|^{p}}{\prod_{j=1}^{m}\left|\Delta^{\left(\beta_{j}+\frac{n / r+m n / r}{m}-2-\varepsilon\right) p+2}\left(\frac{\bar{x}+i \bar{y}_{j}-w_{j}}{i}\right)\right|} \\
& \times \Delta^{p \beta_{1}}\left(y_{1}\right) \ldots \Delta^{p \beta_{m}}\left(y_{m}\right) d x d y_{1} \ldots d y_{m} d \zeta d \eta_{1} \ldots d \eta_{m} .
\end{aligned}
$$

By Fubbini's theorem and applying (3.1), (3.2) and Holder's inequality again we have

$$
\begin{aligned}
& \int_{\Omega^{m}} \int_{\mathbb{R}^{n}} \Delta^{\left(-\varepsilon p^{\prime}-2+n /(r m)+m n / r\right) p / p^{\prime}+\tau_{1}}\left(\eta_{1}\right) \times \cdots \times \Delta^{\left(-\varepsilon p^{\prime}-2+n /(r m)+m n / r\right) p / p^{\prime}+\tau_{m}}\left(\eta_{m}\right) \\
& \times \frac{1}{\prod_{j=1}^{m}\left|\Delta^{\left(\beta_{j}+\frac{n / r+m n / r}{m}-2-\varepsilon\right) p+2}\left(\frac{\bar{x}+i \bar{y}_{j}-w_{j}}{i}\right)\right|} d \zeta d \eta_{1} \ldots d \eta_{m} \\
\leq c_{2} & \prod_{j=1}^{m} \Delta^{\tau_{j}-p \beta_{j}}\left(y_{j}\right) ; \quad y_{j} \in \Omega, j=1, \ldots, m,
\end{aligned}
$$

where $\beta_{j}$ and $\tau_{j}$ satisfy the conditions of the theorem statement.

So

$I(h) \leq c_{3} \int_{\Omega^{m}} \int_{\mathbb{R}^{n}}\left|h\left(x+i y_{j}, \ldots, x+i y_{j}\right)\right|^{p} \Delta^{\tau_{1}}\left(y_{1}\right) \times \cdots \times \Delta^{\tau_{m}}\left(y_{m}\right) d x d y_{1} \ldots d y_{m}$.

The proof of the theorem is complete. 
Note assertions related to boundedness of Bergman-type operators on products of tubular domains over symmetric cones very similar to Theorem 4.1 and 4.2 have direct applications in problems connected with traces in Bergman type analytic function spaces and to various related problems in operator theory. We refer the reader to recent paper [30] and references there and also separately to [4, 23, 24].

We also remark similar assertions based on same technique are valid in more general analytic Bergman type mixed norm spaces on product of tubular domains over symmetric cones. We refer the reader to $[27,28,30]$ for other such type results in analytic mixed norm spaces in context of tubular domains over symmetric cones and also in the unit polyball.

Next let $1 \leq p<\infty, f=f\left(z_{1}, \ldots, z_{m}\right)$, we consider analytic subspaces of $H\left(T_{\Omega}^{m}\right)$, $T_{\Omega}^{m}=T_{\Omega} \times \cdots \times T_{\Omega}, \nu_{j}>\frac{n}{r}-1, \nu>\frac{n}{r}-1, j=1, \ldots, m$. These are spaces $\left(A_{\nu}^{p}\right)_{1}$, $\left(A_{\nu}^{p}\right)_{2},\left(A_{\nu}^{p}\right)_{3}$ with norms

$$
\begin{aligned}
\|f\|_{\left(A_{\vec{\nu})_{1}}^{p}\right.}^{p} & =\int_{T_{\Omega}} \cdots \int_{T_{\Omega}}\left|f\left(x_{1}+i y_{1}, \ldots, x_{m}+i y_{m}\right)\right|^{p} \prod_{j=1}^{m} \Delta^{\nu_{j}-\frac{n}{r}}\left(y_{j}\right) d x_{j} d y_{j}<\infty, \\
\|f\|_{\left(A_{\nu}^{p}\right)_{2}}^{p} & =\int_{\mathbb{R}^{n}} \cdots \int_{\mathbb{R}^{n}} \int_{\Omega}\left|f\left(x_{1}+i y, \ldots, x_{m}+i y\right)\right|^{p} \Delta^{\nu-\frac{n}{r}}(y)\left(\prod_{j=1}^{m} d x_{j}\right) d y<\infty, \\
\|f\|_{\left(A_{\vec{\nu})_{3}}^{p}\right.}^{p} & =\int_{\mathbb{R}^{n}} \int_{\Omega} \ldots \int_{\Omega}\left|f\left(x+i y_{1}, \ldots, x+i y_{m}\right)\right|^{p} \prod_{j=1}^{m} \Delta^{\nu_{j}-\frac{n}{r}}\left(y_{j}\right) d x d y_{j}<\infty .
\end{aligned}
$$

An estimate relating $\|f\|_{\left(A_{\nu}^{p}\right)_{1}}$ and $\|f\|_{A_{\nu}^{p}}$ via Bergman type operator can be seen in recent paper [30].

The natural problem which obviously arise here is to to find some relation between these norms and to find appropriate embeddings between these spaces $\left(A_{\vec{\nu}}^{p}\right)_{1},\left(A_{\vec{\nu}}^{p}\right)_{2}$, $\left(A_{\vec{\nu}}^{p}\right)_{3}$. Note in the unit disk this problem was solved in [27,28].

Let also for $g \in L^{1}\left(T_{\Omega}^{m}\right)$

$$
\begin{aligned}
& \left(V_{\vec{\alpha}, \vec{\beta}} g\right)(\vec{w}) \\
& =\underbrace{\int_{\Omega} \cdots \int_{\Omega}}_{m} \int_{\mathbb{R}^{n}} \frac{g\left(x+i y_{1}, \ldots, x+i y_{m}\right)\left(\Delta y_{1}\right)^{\beta_{1}} \times \cdots \times\left(\Delta y_{m}\right)^{\beta_{m}}}{\prod_{j=1}^{m} \Delta^{\alpha_{j}}\left(\frac{\bar{x}+i \bar{y}_{j}-w_{j}}{i}\right)} d x d y_{1} \ldots d y_{m}, \\
& \left(U_{\vec{\alpha}, \beta} g\right)(\vec{w}) \\
& =\underbrace{\int_{\mathbb{R}^{n}} \ldots \int_{\mathbb{R}^{n}}}_{m} \int_{\Omega} \frac{g\left(x_{1}+i y, \ldots, x_{m}+i y\right)(\Delta y)^{\beta}}{\prod_{j=1}^{m} \Delta^{\alpha_{j}}\left(\frac{\bar{x}+i \bar{y}_{j}-w_{j}}{i}\right)} d y d x_{1} \ldots d x_{m},
\end{aligned}
$$

$w=\left(w_{1}, \ldots, w_{m}\right), \omega_{j} \in T_{\Omega}, j=1, \ldots, m, \beta_{j}>\frac{n}{r}-1, \beta>\frac{n}{r}-1, \alpha_{j}>0, j=1, \ldots, m$, $\vec{\beta}=\left(\beta_{1}, \ldots, \beta_{m}\right)$ or $\vec{\beta}=(\beta, \ldots, \beta)$. 
Let also for $g \in L^{1}\left(T_{\Omega}^{m}\right)$

$$
\left[G_{\vec{\alpha}, \beta}(g)\right]\left(x+i y_{1}, \ldots, x+i y_{m}\right)=\int_{T_{\Omega}} \frac{g(w)\left[\Delta^{\beta}(\operatorname{Im} w)\right] d v(w)}{\left|\prod_{j=1}^{m} \Delta^{\alpha_{j}}\left(\frac{w-\left(\bar{x}+\bar{y}_{j}\right)}{i}\right)\right|},
$$

where $x \in \mathbb{R}^{n}, y_{j} \in \Omega, j=1, \ldots, m, \alpha_{j}>0, \beta>\frac{n}{r}-1, j=1, \ldots, m$.

$$
\left[\tilde{G}_{\vec{\alpha}, \vec{\beta}}(g)\right]\left(x_{1}+i y, \ldots, x_{m}+i y\right)=\int_{T_{\Omega}} \frac{g(w)\left[\Delta^{\beta}(\operatorname{Im} w)\right] d v(w)}{\left|\prod_{j=1}^{m} \Delta^{\alpha_{j}}\left(\frac{w-\left(\bar{x}_{j}+i \bar{y}\right)}{i}\right)\right|},
$$

where $\beta>\frac{n}{r}-1, j=1, \ldots, m ; \alpha_{j}>0, x_{j} \in \mathbb{R}^{n}, j=1, \ldots, m, y \in \Omega$.

The analogue of this theorem in the unit disk can be found in [27].

Theorem 4.3. For $1 \leq p<\infty$, some $\alpha_{j} \in\left(\alpha_{0}, \alpha_{0}^{\prime}\right) ; \beta_{j} \in\left(\beta_{0}, \beta_{0}^{\prime}\right), \beta \in\left(\widetilde{\beta}_{0}, \widetilde{\beta}_{0}^{\prime}\right)$, $\nu_{j}>\left(\frac{n}{r}-1\right), \tau_{j}>\left(\frac{n}{r}-1\right), j=1, \ldots, m$, for some fixed positive $\alpha_{0}^{j},\left(\alpha_{0}^{j}\right)^{\prime}, \beta_{0}^{j},\left(\beta_{0}^{j}\right)^{\prime}$, $j=1, \ldots, m$.

The following estimates are valid:

1) $\left\|G_{\vec{\alpha}, \beta}(g)\right\|_{\left(A_{\vec{\nu}}^{p}\right)_{3}} \leq c_{1}\|g\|_{\left(A_{\tau}^{p}\right)\left(T_{\Omega}\right)}$; for some values $\vec{\nu}$ and $\tau$,

2) $\left\|\tilde{G}_{\vec{\alpha}, \beta}(g)\right\|_{\left(A_{\nu}^{p}\right)_{2}} \leq c_{2}\|g\|_{\left(A_{\tau}^{p}\right)\left(T_{\Omega}\right)}$; for some values $\nu$ and $\tau$,

3) $\left\|V_{\vec{\alpha}, \vec{\beta}}(g)\right\|_{\left(A_{\nu}^{p}\right)_{1}} \leq c_{3}\|g\|_{\left(A_{\vec{\tau}}^{p}\right)_{3}\left(T_{\Omega}\right)}$; for some values $\nu$ and $\vec{\tau}$,

4) $\left\|U_{\vec{\alpha}, \vec{\beta}}(g)\right\|_{\left(A_{\nu}^{p}\right)_{1}} \leq c_{4}\|g\|_{\left(A_{\vec{\tau}}^{p}\right)_{2}\left(T_{\Omega}\right)}$; for some values $\nu$ and $\vec{\tau}$.

where $\alpha_{0}^{j}, \ldots,\left(\beta_{0}^{j}\right)^{\prime}$ depend on $\nu_{j}, \tau_{j}, p, n, \nu, \tau, j=1, \ldots, m$ and $\frac{1}{p}+\frac{1}{p^{\prime}}=1$.

Remark 4.1. Note the following equalities are valid for $G$ and $\tilde{G}$ operators if $-\sum_{j=1}^{m} \nu_{j}+$ $\tau=\beta\left(1+\frac{p}{p^{\prime}}\right)-p \sum_{j=1}^{m} \alpha_{j}+\frac{n}{r}\left(1+\frac{2 p}{p^{\prime}}\right)$ (for $G$ operator) and $-\nu+\tau=\beta\left(1+\frac{p}{p^{\prime}}\right)-$ $p \sum_{j=1}^{m} \alpha_{j}+n / r\left(m+\frac{2 p}{p^{\prime}}\right)$ (for $\tilde{G}$ operator).

Remark 4.2. Note such results may be true for all types of domains of tube type where analogue of Lemma 3.4 can be found.

Remark 4.3. For less general case where $\mathbb{R}^{n}$ is unit interval $I, I=(0,1)$ and $\Omega$ is unit circle $T=\{|z|=1\}$ such type results where proved previously in [27,28].

Proof. We can now prove Theorem 4.3. Let us prove the first estimate. Let

$$
\left[G_{\vec{\alpha}, \beta}(g)\right]\left(x+i y_{1}, \ldots, x+i y_{m}\right)=\int_{T_{\Omega}} \frac{g(w)\left[\Delta^{\beta}(\operatorname{Im} w)\right] d v(w)}{\left|\prod_{j=1}^{m} \Delta^{\alpha_{j}}\left(\frac{w-\left(\bar{x}+\bar{y}_{j}\right)}{i}\right)\right|}
$$

we need to prove that

$$
J=\int_{\mathbb{R}^{n}} \int_{\Omega} \cdots \int_{\Omega}\left|\int_{T_{\Omega}} \frac{g(w)\left[\Delta^{\beta}(\operatorname{Im} w)\right] d v(w)}{\prod_{j=1}^{m} \Delta^{\alpha_{j}}\left(\frac{w-\left(\bar{x}+\bar{y}_{j}\right)}{i}\right)}\right|^{p} \prod_{j=1}^{m} \Delta^{\nu_{j}-\frac{n}{r}}\left(y_{j}\right) d y_{j} d x \leq c\|g\|_{\left(A_{\tau}^{p}\right)\left(T_{\Omega}\right)} .
$$

With some restriction on parameters using Holder's inequality we have

$$
\left.\mid G_{\vec{\alpha}, \beta}(g)\right]\left.\left(x+i y_{1}, \ldots, x+i y_{m}\right)\right|^{p}
$$




$$
\leq \int_{T_{\Omega}} \frac{|g(w)|^{p} \Delta^{\beta}(\operatorname{Im} w) d v(w)}{\prod_{j=1}^{m}\left|\Delta^{p h_{j}}\left(\frac{w-\left(\bar{x}+i \bar{y}_{j}\right)}{i}\right)\right|}\left(\int_{T_{\Omega}} \frac{\Delta^{\beta}(\operatorname{Im} w) d v(w)}{\prod_{j=1}^{m}\left|\Delta^{p^{\prime} b_{j}}\left(\frac{w-\left(\bar{x}+i \bar{y}_{j}\right)}{i}\right)\right|}\right)^{\frac{p}{p^{\prime}}}
$$

where $h_{j}+b_{j}=\alpha_{j}, \frac{1}{p}+\frac{1}{p^{\prime}}=1, j=1, \ldots, m$.

Let us estimate the second integral using the third estimate of Lemma 3.4

$$
\begin{aligned}
\left(\int_{T_{\Omega}} \frac{\Delta^{\beta}(\Im w) d v(w)}{\prod_{j=1}^{m}\left|\Delta^{p^{\prime} b_{j}}\left(\frac{w-\left(\bar{x}+i \bar{y}_{j}\right)}{i}\right)\right|}\right)^{\frac{p}{p^{\prime}}} & \leq \prod_{j=1}^{m}\left(\int_{T_{\Omega}} \frac{\Delta^{\beta}(\operatorname{Im} w) d v(w)}{\left|\Delta^{p^{\prime} m b_{j}}\left(\frac{w-\left(\bar{x}+i \bar{y}_{j}\right)}{i}\right)\right|}\right)^{\frac{p}{m p^{\prime}}} \\
& \leq c_{1} \prod_{j=1}^{m} \Delta^{\left(\beta-p^{\prime} m b_{j}+2 n / r\right) \frac{p}{m p^{\prime}}}\left(y_{j}\right) .
\end{aligned}
$$

Denote $\left(\beta / m-p^{\prime} b_{j}+2 n /(m r)\right) \frac{p}{p^{\prime}}=-\nu_{j}+\frac{n}{r}, j=1, \ldots, m$. In this case we have

$$
\begin{aligned}
I & =\int_{\mathbb{R}^{n}}\left|\int_{T_{\Omega}} \frac{g(w) \Delta^{\beta}(\operatorname{Im} w) d v(w)}{\prod_{j=1}^{m} \Delta^{\alpha_{j}}\left(\frac{w-\left(\bar{x}+i \bar{y}_{j}\right)}{i}\right)}\right|^{p} \prod_{j=1}^{m} \Delta^{\nu_{j}-\frac{n}{r}}\left(y_{j}\right) d x \\
& \leq c_{1} \int_{\mathbb{R}^{n}} \int_{T_{\Omega}} \frac{|g(w)|^{p} \Delta^{\beta}(\operatorname{Im} w) d v(w)}{\prod_{j=1}^{m}\left|\Delta^{p h_{j}}\left(\frac{w-\left(\bar{x}+i \bar{y}_{j}\right)}{i}\right)\right|} d x .
\end{aligned}
$$

Using Fubbini's theorem we obtain

$$
I \leq c_{1} \int_{T_{\Omega}}|g(w)|^{p} \Delta^{\beta}(\operatorname{Im} w) \prod_{j=1}^{m}\left(\int_{\mathbb{R}^{n}} \frac{d x}{\left|\Delta^{m p h_{j}}\left(\frac{w-\left(\bar{x}+i \bar{y}_{j}\right)}{i}\right)\right|}\right)^{\frac{1}{m}} d v(w) .
$$

From (3.2) we have

$$
I \leq c_{2} \int_{T_{\Omega}}|g(w)|^{p} \Delta^{\beta}(\operatorname{Im} w) \prod_{j=1}^{m} \frac{1}{\left|\Delta^{p h_{j}-n /(m r)}\left(\frac{\operatorname{Im} w-i \bar{y}_{j}}{i}\right)\right|} d v(w) .
$$

So

$$
J \leq c_{2} \int_{\Omega} \ldots \int_{\Omega} \int_{T_{\Omega}}|g(w)|^{p} \Delta^{\beta}(\operatorname{Im} w) \prod_{j=1}^{m} \frac{1}{\left|\Delta^{p h_{j}-n /(m r)}\left(\frac{\operatorname{Im} w-i \bar{y}_{j}}{i}\right)\right|} d v(w) d y_{1} \ldots d y_{m} .
$$

Next using Fubbini theorem and Lemma 3.4 we have

$$
\begin{aligned}
& J \leq c_{2} \int_{T_{\Omega}}|g(w)|^{p} \Delta^{\beta}(\operatorname{Im} w) \prod_{j=1}^{m} \int_{\Omega} \frac{1}{\left|\Delta^{p h_{j}-n /(m r)}\left(\frac{\operatorname{Im} w-i \bar{y}_{j}}{i}\right)\right|} d y_{j} d v(w), \\
& J \leq c_{3} \int_{T_{\Omega}}|g(w)|^{p} \Delta^{\beta-p \sum_{j=1}^{m} h_{j}+m n / r+n / r}(\operatorname{Im} w) d v(w)=\int_{T_{\Omega}}|g(w)|^{p} \Delta^{\tau}(\operatorname{Im} w) d v(w),
\end{aligned}
$$


where $\tau=\beta-p \sum_{j=1}^{m} h_{j}+m n / r+n / r$. But $\left(-\sum_{j=1}^{m} \nu_{j}+\frac{m n}{r}\right)=\beta p / p^{\prime}-p \sum_{j=1}^{m} b_{j}+$ $2 n p /\left(r p^{\prime}\right)$ and $-\sum_{j=1}^{m} \nu_{j}+\tau=\beta\left(1+\frac{p}{p^{\prime}}\right)-p \sum_{j=1}^{m} \alpha_{j}+\frac{n}{r}\left(1+\frac{2 p}{p^{\prime}}\right)$.

We prove the second case now. Let

$$
\left[\tilde{G}_{\vec{\alpha}, \beta}(g)\right]\left(x_{1}+i y, \ldots, x_{m}+i y\right)=\int_{T_{\Omega}} \frac{g(w) \Delta^{\beta}(\operatorname{Im} w) d v(w)}{\prod_{j=1}^{m} \Delta^{\alpha_{j}}\left(\frac{w-\left(\bar{x}_{j}+i \bar{y}\right)}{i}\right)} .
$$

We assume that all $\alpha_{j}, j=1, \ldots, m$ are large enough numbers.

We need to prove that

$$
J=\int_{\mathbb{R}^{n}} \cdots \int_{\mathbb{R}^{n}} \int_{\Omega}\left|\int_{T_{\Omega}} \frac{g(w) \Delta^{\beta}(\operatorname{Im} w) d v(w)}{\prod_{j=1}^{m} \Delta^{\alpha_{j}}\left(\frac{w-\left(\bar{x}_{j}+i \bar{y}\right)}{i}\right)}\right|^{p} \Delta^{\nu-\frac{n}{r}}(y) d y\left(\prod_{j=1}^{m} d x_{j}\right) \leq c\|g\|_{\left(A_{\tau}^{p}\right)\left(T_{\Omega}\right)} .
$$

With some restriction on parameters using Holder's inequality we have

$$
\begin{aligned}
\left.\mid \tilde{G}_{\vec{\alpha}, \beta}(g)\right]\left.\left(x_{1}+i y, \ldots, x_{m}+i y\right)\right|^{p} \leq & \int_{T_{\Omega}} \frac{|g(w)|^{p} \Delta^{\beta}(\operatorname{Im} w) d v(w)}{\prod_{j=1}^{m}\left|\Delta^{p h_{j}}\left(\frac{w-\left(\bar{x}_{j}+i \bar{y}\right)}{i}\right)\right|} \\
& \times\left(\int_{T_{\Omega}} \frac{\Delta^{\beta}(\operatorname{Im} w) d v(w)}{\prod_{j=1}^{m}\left|\Delta^{p^{\prime} b_{j}}\left(\frac{w-\left(\bar{x}_{j}+i \bar{y}\right)}{i}\right)\right|}\right)^{\frac{p}{p^{\prime}}},
\end{aligned}
$$

where $h_{j}+b_{j}=\alpha_{j}, \frac{1}{p}+\frac{1}{p^{\prime}}=1, j=1, \ldots, m$.

Let us estimate the second integral using third part of Lemma 3.4

$$
\begin{aligned}
\left(\int_{T_{\Omega}} \frac{\Delta^{\beta}(\operatorname{Im} w) d v(w)}{\left.\prod_{j=1}^{m} \mid \Delta^{p^{\prime} b_{j}\left(\frac{w-\left(\bar{x}_{j}+i \bar{y}\right)}{i}\right) \mid}\right)^{\frac{p}{p^{\prime}}}}\right. & \leq \prod_{j=1}^{m}\left(\int_{T_{\Omega}} \frac{\Delta^{\beta}(\operatorname{Im} w) d v(w)}{\left|\Delta^{p^{\prime} m b_{j}}\left(\frac{w-\left(\bar{x}_{j}+i \bar{y}\right)}{i}\right)\right|}\right)^{\frac{p}{m p^{\prime}}} \\
& \leq c_{1} \prod_{j=1}^{m} \Delta^{\left(\beta-p^{\prime} m b_{j}+2 n / r\right) \frac{p}{m p^{\prime}}}(y) \\
& =c_{1} \Delta^{\left(\beta-p^{\prime} \sum_{j=1}^{m} b_{j}+2 n / r\right) \frac{p}{p^{\prime}}}(y)
\end{aligned}
$$

Denote $\left(\beta-p^{\prime} \sum_{j=1}^{m} b_{j}+2 n / r\right) \frac{p}{p^{\prime}}=-\nu+\frac{n}{r}$. In this case we have

$$
\begin{aligned}
I & =\int_{\mathbb{R}^{n}} \cdots \int_{\mathbb{R}^{n}}\left|\int_{T_{\Omega}} \frac{g(w) \Delta^{\beta}(\operatorname{Im} w) d v(w)}{\prod_{j=1}^{m} \Delta^{\alpha_{j}}\left(\frac{w-\left(\bar{x}_{j}+i \bar{y}\right)}{i}\right)}\right|^{p} \Delta^{\nu-\frac{n}{r}}(y)\left(\prod_{j=1}^{m} d x_{j}\right) \\
& \leq c_{1} \int_{\mathbb{R}^{n}} \cdots \int_{\mathbb{R}^{n}} \int_{T_{\Omega}} \frac{|g(w)|^{p} \Delta^{\beta}(\operatorname{Im} w) d v(w)}{\prod_{j=1}^{m}\left|\Delta^{p h_{j}}\left(\frac{w-\left(\bar{x}_{j}+i \bar{y}\right)}{i}\right)\right|}\left(\prod_{j=1}^{m} d x_{j}\right) .
\end{aligned}
$$


Using Fubbini's theorem we obtain

$$
I \leq c_{1} \int_{T_{\Omega}}|g(w)|^{p} \Delta^{\beta}(\operatorname{Im} w) \int_{\mathbb{R}^{n}} \cdots \int_{\mathbb{R}^{n}} \frac{1}{\prod_{j=1}^{m}\left|\Delta^{p h_{j}}\left(\frac{\operatorname{Im} w-\left(\bar{x}_{j}+i \bar{y}\right)}{i}\right)\right|}\left(\prod_{j=1}^{m} d x_{j}\right) d v(w) .
$$

From Lemma 3.4 we have for large enough $h_{j}, j=1, \ldots, m$

$$
I \leq c_{2} \int_{T_{\Omega}}|g(w)|^{p} \frac{\Delta^{\beta}(\operatorname{Im} w)}{\left|\Delta^{\sum_{j=1}^{m} p h_{j}-m n / r}\left(\frac{\operatorname{Im} w-i \bar{y}}{i}\right)\right|} d v(w) .
$$

So

$$
J \leq c_{2} \int_{\Omega} \int_{T_{\Omega}}|g(w)|^{p} \frac{\Delta^{\beta}(\operatorname{Im} w)}{\left|\Delta^{p \sum_{j=1}^{m} h_{j}-m n / r}\left(\frac{\operatorname{Im} w-i \bar{y}}{i}\right)\right|} d v(w) d y .
$$

Next using Fubbini's theorem and Lemma 3.4 we have

$$
\begin{aligned}
J & \leq c_{2} \int_{T_{\Omega}}|g(w)|^{p} \Delta^{\beta}(\operatorname{Im} w) \int_{\Omega} \frac{1}{\left|\Delta^{p \sum_{j=1}^{m} h_{j}-m n / r}\left(\frac{\operatorname{Im} w-i \bar{y}}{i}\right)\right|} d y d v(w), \\
J & \leq c_{3} \int_{T_{\Omega}}|g(w)|^{p} \Delta^{\beta-p \sum_{j=1}^{m} h_{j}+m n / r+n / r}(\operatorname{Im} w) d v(w) \\
& =c_{3} \int_{T_{\Omega}}|g(w)|^{p} \Delta^{\tau}(\operatorname{Im} w) d v(w),
\end{aligned}
$$

where $\tau=\beta-p \sum_{j=1}^{m} h_{j}+m n / r+n / r$. But $\left(-\nu+\frac{n}{r}\right)=\beta \frac{p}{p^{\prime}}-p \sum_{j=1}^{m} b_{j}+2 n / r \frac{p}{p^{\prime}}$ and $-\nu+\tau=\beta\left(1+\frac{p}{p^{\prime}}\right)-p \sum_{j=1}^{m} \alpha_{j}+n / r\left(m+\frac{2 p}{p^{\prime}}\right)$.

We omit proofs of the third and forth cases because they are similar. The proof of theorem is complete.

Remark 4.4. As a direct corollary of Whitney decomposition of $\Omega$ cone and Theorem 4.3 we have the following estimate

$$
\begin{aligned}
\int_{\Omega} \int_{\mathbb{R}^{n}}|g(\vec{w})|^{p} \Delta(\operatorname{Im} w)^{\alpha-\frac{n}{r}} d v(w) \leq & \underbrace{\int_{\Omega} \ldots \int_{\Omega}}_{m} \int_{\mathbb{R}^{n}}\left|g\left(x_{1}+i y_{1}, \ldots, x_{m}+i y_{m}\right)\right|^{p} \\
& \times \prod_{j=1}^{m} \Delta\left(\operatorname{Im} w_{j}\right)^{\alpha_{j}} d v\left(w_{j}\right),
\end{aligned}
$$

where $w_{j}=x+i y_{j}, j=1, \ldots, m, g \in H\left(T_{\Omega}^{m}\right), \alpha_{j} \in(\tilde{\alpha}, \infty), \alpha \in\left(\tilde{\alpha}_{0}, \infty\right), \alpha>\frac{n}{r}-1$, $\alpha_{j}>\frac{n}{r}-1, j=1, \ldots, m, 0<p<\infty$ for some fixed $\tilde{\alpha}$ and $\tilde{\alpha}_{0}$, where $\alpha$ depends on all $\alpha_{j}$.

The proof of this estimate repeats arguments provided in one dimensional case of unit disk in [27] which is also based on Whitney decomposition of unit interval $(0,1)$ and the fact that inner integral is monotonic in cone (see [3, page 30]). 
Similar estimate

$$
\begin{aligned}
\int_{\Omega} \int_{\mathbb{R}^{n}}|g(\vec{w})|^{p} \Delta(\operatorname{Im} w)^{\alpha-\frac{n}{r}} d v(w) \leq c_{1} & \int_{T_{\Omega}} \ldots \int_{T_{\Omega}}\left|g\left(x+i y_{1}, \ldots, x+i y_{m}\right)\right|^{p} \\
& \times \prod_{j=1}^{m} \Delta\left(\operatorname{Im} w_{j}\right)^{\alpha_{j}-\frac{n}{r}} d v\left(w_{j}\right)
\end{aligned}
$$

where $1<p<\infty, \alpha_{j}>\frac{n}{r}-1, \alpha>\frac{n}{r}-1, j=1, \ldots, m$, where $\alpha$ parameter depends on all $\alpha_{j}, j=1, \ldots, m$ was obtained recently in [30].

Both assertions for unit ball can be seen in [28] and [26].

\section{Multifunctional Analytic Spaces in Tubular Domains Over Symmetric Cones.}

The intention of this section is to introduce for the first time in literature multifunctional analytic spaces in tubular domains over symmetric cone and provide their basic properties.

Similar results for harmonic function spaces and for analytic spaces in the unit ball can be seen in $[1,17]$.

We will need for all proofs various properties of $r$-lattice of $T_{\Omega}$ (see for example [24]) and various nice properties of Bergman balls from recent papers [3,4]). We listed all these properties in detail in previous section.

Theorem 5.1. Let $\alpha>-1, F_{i} \in H\left(T_{\Omega} \times \cdots \times T_{\Omega}\right), i=1, \ldots, m, T_{\Omega}^{t}=T_{\Omega} \times \cdots \times T_{\Omega}$, $t \in \mathbb{N}$. Let $0<p_{i}, q_{i}<\infty, i=1, \ldots, m$ so $\sum_{i=1}^{m} \frac{p_{i}}{q_{i}}=1$. Then we have

$$
\begin{aligned}
& \int_{T_{\Omega}}\left|F_{1}(w, \ldots, w)\right|^{p_{1}} \times \cdots \times\left|F_{m}(w, \ldots, w)\right|^{p_{m}} \Delta^{\alpha}(\operatorname{Im} w) d \nu(w) \\
\leq & c \prod_{i=1}^{m}\left(\int_{T_{\Omega}} \ldots \int_{T_{\Omega}}\left|F_{i}\left(w_{1}, \ldots, w_{t}\right)\right|^{q_{i}} \prod_{j=1}^{t} \Delta^{\beta_{i}}\left(\operatorname{Im} w_{j}\right) d v\left(w_{j}\right)\right)^{\frac{p_{i}}{q_{i}}},
\end{aligned}
$$

where

$$
\beta_{i}=\frac{\left(\frac{2 n}{r}+\alpha\right) q_{i}}{t m p_{i}}-\frac{2 n}{r}>-1, \quad i=1, \ldots, m .
$$

If all $p_{j}=q_{j}=p, j=1, \ldots, m$ above then we get the "limit case" of Theorem 5.1.

Theorem 5.2. Let $F_{i} \in H\left(T_{\Omega} \times \cdots \times T_{\Omega}\right), i=1, \ldots, m, T_{\Omega}^{t}=T_{\Omega} \times \cdots \times T_{\Omega}, t \in \mathbb{N}$, $\alpha>-1, \alpha>$ tn $-n-1, \beta_{i}>-1,0<p<\infty$. Then

$$
\int_{T_{\Omega}} \prod_{i=1}^{m}\left|F_{i}(w, \ldots, w)\right|^{p} \Delta^{\alpha}(\operatorname{Im} w) d v(w)
$$




$$
\leq c \int_{T_{\Omega}} \ldots \int_{T_{\Omega}} \prod_{i=1}^{m}\left|F_{i}\left(w_{1}, \ldots, w_{t}\right)\right|^{p} \prod_{j=1}^{t} \Delta^{\beta_{j}}\left(\operatorname{Im} w_{j}\right) d v\left(w_{j}\right)
$$

where

$$
\beta_{i}=\frac{\frac{2 n}{r}+\alpha}{t}-\frac{2 n}{r}, \quad i=1, \ldots, t
$$

Remark 5.1. Note for $t=1, m=1$ these estimates are obvious. For $m=1, t>1$ in case of unit disk, polydisk these estimates can be found in [25,27]. For case of unit ball $D=B^{n} \subset \mathbb{C}^{n}$ these results can be found in [17].

Remark 5.2. For case of unit ball $B \subset \mathbb{C}^{n}$ these results can be found in [17]. For $m=1$, $t>1$ this result for unit ball can be found in [17]. For unit disk $D=\{z \in \mathbb{C}:|z|<1\}$ these estimates were found much earlier by various authors (see, for example, $[25,33]$ and references there).

Let $\mu$ be a measure supported in the boundary of the cone. M. Vergne and H. Rossi $([13,31])$ studied analytic function spaces of Hardy type

$$
H_{\mu}^{p}=\left\{F \in H\left(T_{\Omega}\right): \sup _{y \in \Omega} \int_{\partial \Omega} \int_{\mathbb{R}^{n}}|F(x+i(y+t))|^{p} d x d \mu(t)<\infty\right\} .
$$

Similar spaces will be considered by us below.

\section{Theorem 5.3.}

1) Let $\mu$ be a positive Borel measure on $T_{\Omega}$ and let $\left\{a_{k}\right\}$ be a sequence an $r$-lattice based on Bergman balls. Let $f_{j} \in H\left(T_{\Omega}\right), j=1, \ldots, m, 0<p_{i}, q_{i}<\infty, i=1, \ldots, m+1$, $\sum_{i=1}^{m+1} \frac{p_{i}}{q_{i}}=1$. If

$$
\left(\sum_{k=1}^{\infty}\left(\int_{B_{T_{\Omega}}\left(a_{k}\right)} d \mu(z)\right)^{\frac{q_{m+1}}{p_{m+1}}}\right)^{\frac{p_{m+1}}{q_{m+1}}} \leq c
$$

then we have the following estimate

$$
\int_{T_{\Omega}} \prod_{i=1}^{m}\left|f_{i}(z)\right|^{p_{i}} \Delta(\operatorname{Im} z)^{m\left(\frac{2 n}{r}\right)} d \mu(z) \leq c\left[\prod_{i=1}^{m}\left(\sum_{k=1}^{\infty}\left(\int_{B_{T_{\Omega}}\left(a_{k}\right)}\left|f_{i}(z)\right|^{p_{i}} d v(z)\right)^{\frac{q_{i}}{p_{i}}}\right)^{\frac{p_{i}}{q_{i}}}\right] .
$$

2) Let $\mu$ be a positive Borel measure on $T_{\Omega}$ and let $\left\{a_{k}\right\}$ be a sequence an $r$-lattice based on Bergman balls. Let $f_{j} \in H\left(T_{\Omega}\right), j=1, \ldots, m, 0 \leq p_{i}<q_{i}<\infty, i=1, \ldots, m+1$, 
so that $\sum_{i=1}^{m+1} \frac{p_{i}}{q_{i}}=1$. If

$$
\left(\sum_{k=1}^{\infty}\left(\int_{B_{T_{\Omega}}\left(a_{k}\right)} d \mu(z)\right)^{\frac{q_{m+1}}{p_{m+1}}}\right)^{\frac{p_{m+1}}{q_{m+1}}} \leq c<\infty
$$

then we have following estimate

$$
\int_{T_{\Omega}} \prod_{i=1}^{m}\left|f_{i}(z)\right|^{p_{i}}(\Delta(\operatorname{Im} z))^{\left(\frac{2 n}{r}\right) \sum_{i=1}^{m} \frac{p_{i}}{q_{i}}} d \mu(z) \leq c \prod_{i=1}^{m}\left(\int_{T_{\Omega}}\left|f_{i}(z)\right|^{q_{i}} d v(z)\right)^{\frac{p_{i}}{q_{i}}}
$$

Below based on preliminaries we provided complete proofs of our assertions (Theorems 5.1-5.3) will be given, some proofs however are missed. We refer the reader for them to [17], where analogues for unit ball can be found. The main idea is the adaptation of $r$-lattice of $T_{\Omega}$ to $r$-lattice of unit ball and we leave this partially to readers.

Various results on product domains can be seen in [15] and for other product domains in [7]. Hence our results also can be seen as some supplement of results from [15] and [7]. All these results can be seen also as direct extensions of estimates previously known in polydisk which is the simple case.

Note again our proofs are parallel to the unit ball case and we will omit some of them here. We again should heavily use in all proofs certain nice properties of $r$-lattices in tubular domains over symmetric cones which can be seen for example in [24]. In the case of unit ball we heavily used similar properties of $r$-lattice, but for unit ball (see [17]), moreover our arguments are similar.

Let us prove of Theorem 5.1 now.

Proof. Let $\left\{a_{k}\right\}$ be an $r$-lattice based on $B_{T_{\Omega}}\left(a_{k}, R\right)=B_{T_{\Omega}}\left(a_{k}\right)$ Bergman balls. Using properties we listed above we have:

$$
\begin{aligned}
I & =\int_{T_{\Omega}} \prod_{i=1}^{m}\left|F_{i}(w, \ldots, w)\right|^{p_{i}} \Delta^{\alpha}(\operatorname{Im} w) d v(w) \\
& \leq c \sum_{k=1}^{\infty} \int_{B_{T_{\Omega}}\left(a_{k}\right)} \prod_{i=1}^{m}\left|F_{i}(w, \ldots, w)\right|^{p_{i}} \Delta^{\alpha}(\operatorname{Im} w) d v(w) \\
& \leq c_{1} \sum_{k=1}^{\infty} \sup _{z \in B_{T_{\Omega}}\left(a_{k}\right)} \prod_{i=1}^{m}\left|F_{i}(z, \ldots, z)\right|^{p_{i}} \Delta^{\alpha}\left(\operatorname{Im} a_{k}\right) v\left(B_{T_{\Omega}}\left(a_{k}\right)\right)
\end{aligned}
$$




$$
\begin{aligned}
& \leq c_{2} \sum_{k_{1}=1}^{\infty} \cdots \sum_{k_{t}=1}^{\infty}\left[\sup _{z_{1} \in B_{T_{\Omega}}\left(a_{k_{1}}\right)}\left|F_{1}\left(z_{1}, \ldots, z_{t}\right)\right|^{p_{1}}\right. \\
& z_{t} \in B_{T_{\Omega}}\left(a_{k_{t}}\right) \\
& \left.\times \cdots \times \sup _{z_{1} \in B_{T_{\Omega}}\left(a_{k_{1}}\right)}\left|F_{m}\left(z_{1}, \ldots, z_{t}\right)\right|^{p_{m}} \times \ldots\right] \\
& \text { : } \\
& z_{t} \in B_{T_{\Omega}}\left(a_{k_{t}}\right) \\
& \times\left[\Delta^{\frac{2 n}{r}+\alpha}\left(\operatorname{Im} a_{k_{1}}\right) \times \cdots \times \Delta^{\frac{\frac{2 n}{r}+\alpha}{t}}\left(\operatorname{Im} a_{k_{t}}\right)\right] .
\end{aligned}
$$

Using Holder inequality for $m$-functions and again properties of $r$-lattice we listed above we have

$$
\begin{aligned}
& I \leq c_{4} \prod_{s=1}^{m}\left(\sum_{k_{1}, \ldots, k_{t}=1}^{\infty} \sup _{z_{1} \in B_{T_{\Omega}}\left(a_{k_{1}}\right)}\left|F_{s}\left(z_{1}, \ldots, z_{t}\right)\right|^{q_{s}} \prod_{s=1}^{t} \Delta^{\frac{\left(\frac{2 n}{r}+\alpha\right) q_{s}}{t m p_{s}}}\left(\operatorname{Im} a_{k_{s}}\right)\right)^{\frac{p_{s}}{q_{s}}} \\
& z_{t} \in B_{T_{\Omega}}\left(a_{k_{t}}\right) \\
& \leq c_{5} \prod_{s=1}^{m}\left(\sum_{k_{1}, \ldots, k_{t}=1}^{\infty} \int_{T_{\Omega}\left(a_{k_{1}}, \widetilde{R}\right)} \ldots \int_{T_{\Omega}\left(a_{k_{t}}, \widetilde{R}\right)}\left|F_{s}\left(w_{1}, \ldots, w_{t}\right)\right|^{q_{s}} d v\left(w_{1}\right) \ldots d v\left(w_{t}\right)\right. \\
& \left.\times \prod_{j=1}^{t} \Delta^{\beta_{j}}\left(\operatorname{Im} a_{k_{j}}\right)\right)^{\frac{p_{s}}{q_{s}}} \\
& \leq c_{6}\left(\int_{T_{\Omega}} \ldots \int_{T_{\Omega}}\left|F_{1}\left(w_{1}, \ldots, w_{t}\right)\right|^{q_{1}} \prod_{s=1}^{t}\left(\Delta\left(\operatorname{Im} w_{s}\right)\right)^{\beta_{s}} d v\left(w_{1}\right) \ldots d v\left(w_{t}\right)\right)^{\frac{p_{1}}{q_{1}}} \\
& \times \cdots \times\left(\int_{T_{\Omega}} \cdots \int_{T_{\Omega}}\left|F_{m}\left(w_{1}, \ldots, w_{t}\right)\right|^{q_{m}} \prod_{j=1}^{t}\left(\Delta\left(\operatorname{Im} w_{j}\right)\right)^{\beta_{j}} d v\left(w_{1}\right) \ldots d v\left(w_{t}\right)\right)^{\frac{p_{m}}{q_{m}}}, \\
& \widetilde{R}=\frac{1+R}{2}, \quad R \in(0,1) .
\end{aligned}
$$

The proof of theorem is complete.

Proof of Theorem 5.2. can be obtained by small modification of Theorem 5.1 and we omit here details, referring the reader also to unit ball case (see [17]).

And now we can prove Theorem 5.3. 
Proof. We again follow arguments of unit ball case and properties of $r$-lattice from $[3,4]$ which we listed above, we omit the first part referring to our mentioned paper [17] and concentrate only on proof of second part of our theorem.

Note also the proof of first part has similarities with the proof of second part below. We have the following estimates. Suppose (5.1) holds then we have by Holder inequality

$$
\begin{aligned}
& J=\int_{T_{\Omega}} \prod_{i=1}^{m}\left|f_{i}(z)\right|^{p_{i}}\left(\Delta^{\left(\frac{2 n}{r}\right) \sum_{i=1}^{m} \frac{p_{i}}{q_{i}}}(\operatorname{Im} z)\right) d \mu(z) \\
& =\sum_{k=1}^{\infty} \int_{B_{T_{\Omega}}\left(a_{k}, r\right)}\left(\prod_{i=1}^{m}\left|f_{i}\left(z_{i}\right)\right|^{p_{i}}\right) \Delta^{\tau}(\operatorname{Im} z) d \mu(z) \\
& \leq c \sum_{k=1}^{\infty} \prod_{i=1}^{m} \sup _{z \in B_{T_{\Omega}}\left(a_{k}, r\right)}\left|f_{i}(z)\right|^{p_{i}} \int_{B_{T_{\Omega}}\left(a_{k}, r\right)} \Delta^{\left(\frac{2 n}{r}\right) \sum_{i=1}^{m} \frac{p_{i}}{q_{i}}}(\operatorname{Im} z) d \mu(z) \\
& \leq c_{1}\left(\sum_{k=1}^{\infty} \sup _{z \in B_{T_{\Omega}}\left(a_{k}, r\right)}\left|f_{1}(z)\right|^{q_{1}} \Delta^{\frac{2 n}{r}}(\operatorname{Im} z)\right)^{\frac{p_{1}}{q_{1}}} \\
& \times \cdots \times\left(\sum_{k=1}^{\infty} \sup _{z \in B_{T_{\Omega}}\left(a_{k}, r\right)}\left|f_{m}(z)\right|^{q_{m}} \Delta^{\frac{2 n}{r}}(\operatorname{Im} z)\right)^{\frac{p_{m}}{q_{m}}} \\
& \times\left(\sum_{k=1}^{\infty}\left[\int_{B_{T_{\Omega}}\left(a_{k}, r\right)} d \mu(z)\right]^{\frac{q_{m+1}}{p_{m+1}}}\right)^{\frac{p_{m+1}}{q_{m+1}}} \\
& \leq c_{2} \prod_{i=1}^{m}\left(\int_{T_{\Omega}}\left|f_{i}(z)\right|^{q_{i}} d v(z)\right)^{\frac{p_{i}}{q_{i}}}\left(\sum_{k=1}^{\infty}\left[\int_{B_{T_{\Omega}}\left(a_{k}, r\right)} d \mu(z)\right]^{\frac{q_{m+1}}{p_{m+1}}}\right)^{\frac{p_{m+1}}{q_{m+1}}} \\
& \leq c_{3} \prod_{i=1}^{m}\left(\int_{T_{\Omega}}\left|f_{i}(z)\right|^{q_{i}} d v(z)\right)^{\frac{p_{i}}{q_{i}}} \text {. }
\end{aligned}
$$

Theorem 5.3 is proved.

Finally we add a series of various remarks.

Remark 5.3. We note that various (not sharp) embedding theorems can be obtained from the following simple observation also related with $r$-lattices (we give only one 
function model). We search condition on positive Borel measure $\mu$ so that

$$
\int_{T_{\Omega}}|f(z)|^{p} d \mu(z) \leq c\|f\|_{Y}^{p}
$$

where $Y$ is a holomorphic subspace of $H\left(T_{\Omega}\right)$. We have for any $\left\{a_{k}\right\}-r$-lattice

$$
\begin{aligned}
\int_{T_{\Omega}}|f(z)|^{p} \Delta^{\alpha}(\operatorname{Im} z) d \mu(z) & \leq \sum_{k=1}^{\infty} \max _{z \in B_{T_{\Omega}}\left(a_{k}, r\right)}|f(z)|^{p} \Delta^{\alpha}(\operatorname{Im} z) \mu\left(B_{T_{\Omega}}\left(a_{k}, r\right)\right) \\
& \leq c \int_{T_{\Omega}}|f(z)|^{p} g_{1}(z) \Delta^{\alpha}(\operatorname{Im} z) d v(z)
\end{aligned}
$$

where $g_{1}(z)=\sum_{k=1}^{\infty} \Delta^{-\left(\frac{2 n}{r}\right)}\left(\operatorname{Im} a_{k}\right)\left[\mu\left(B_{T_{\Omega}}\left(a_{k}, r\right)\right)\right]\left[\chi_{B_{T_{\Omega}}\left(a_{k}, r\right)}(z)\right], z \in T_{\Omega}, 0<p<\infty$, $\alpha>-1$.

Remark 5.4. Note also estimates of this section can be partially extended to some mixed norm spaces defined on product domains. For these type spaces in pseudoconvex domains we refer the reader to [20].

We define these mixed norm spaces on product domains as spaces with norms

$$
\int_{\Omega^{m}}(\underbrace{\int_{\mathbb{R}^{n}} \cdots \int_{\mathbb{R}^{n}}}_{m}\left|f\left(z_{1}, \ldots, z_{m}\right)\right| d x_{1} \ldots d x_{m})^{\frac{q}{p}} \prod_{j=1}^{m} \Delta^{\gamma_{j}-\frac{n}{r}}\left(y_{j}\right) d y_{j},
$$

where $1<p<\infty, 1<q \leq \infty, \gamma_{j}>\frac{n}{r}-1, j=1, \ldots, n$.

Note for $m=1$ and $p=q$ we get ordinary Bergman space $A_{\beta}^{p}\left(T_{\Omega}\right)$ with norm

$$
\left(\int_{T_{\Omega}}|f(z)|^{p} \Delta^{\beta}(\operatorname{Im} z) d v(z)\right)^{\frac{1}{p}}, \quad 1<p<\infty, \quad \beta>-1,
$$

and for $m>1, p=q$ we get the Bergman spaces on product domains with the norm

$$
\left(\int_{T_{\Omega}} \cdots \int_{T_{\Omega}}\left|f\left(z_{1}, \ldots, z_{m}\right)\right|^{p} \Delta^{\beta_{1}}\left(\operatorname{Im} z_{1}\right) \times \cdots \times \Delta^{\beta_{m}}\left(\operatorname{Im} z_{m}\right) d v\left(z_{1}\right) \ldots d v\left(z_{m}\right)\right)^{\frac{1}{p}}
$$

where $1<p<\infty, \beta_{j}>-1, j=1, \ldots, m$. At the end we discuss some issues related to integral operators on product of cones.

Remark 5.5. It is known (see [6])

$$
L=\int_{\Omega}\left(\int_{\Omega} f(v+y) \Delta(y)^{m-n / r} d y\right)^{q} \Delta(v)^{\nu-n / r} d v
$$




$$
\begin{aligned}
& \leq c \int_{\Omega}\left(\Delta(y)^{m} f(y)\right)^{q} \Delta^{\nu-n / r} d y \\
& =L_{2},
\end{aligned}
$$

if $1 \leq q<q_{\nu}, f \geq 0, m>n / r-1, \nu>n / r-1, L_{2}<\infty$ for some fixed $q_{\nu}$.

We can consider for $m>n / r-1, f>0, f=f\left(v_{1}, \ldots, v_{k}\right), v_{j} \in \Omega, j=1, \ldots, k$ more general versions

$$
\left(T_{m}^{k} f\right)(\vec{v})=\int_{\Omega} f\left(v_{1}+y, \ldots, v_{k}+y\right) \Delta(y)^{m-n / r} d y, \quad k \in \mathbb{N},
$$

and

$$
\begin{aligned}
&\left(T_{m}^{k} f\right)\left(v_{1}, \ldots, v_{k}\right)=\underbrace{\int_{\Omega} \cdots \int_{\Omega}}_{k} f\left(v_{1}+y_{1}, \ldots, v_{k}+y_{k}\right) \\
& \quad \times \Delta\left(y_{1}\right)^{m_{1}-n / r} \times \cdots \times \Delta^{m_{k}-n / r}\left(y_{k}\right) d y_{1} \ldots d y_{k},
\end{aligned}
$$

$k \in \mathbb{N}, m_{j}>n / r-1, j=1, \ldots, k$ and show analogues of (5.2) as we did with Bergman type integral operators on product domains in previous section.

It is known also (see [6]) the classical Bergman projections are closely related with Hardy type inequalities and some duality theorems in $A_{\tau}^{p, q}$ spaces. We give an example of Hardy type inequality.

$$
\|F\|_{L_{\nu}^{p, q}} \leq c_{p, q}\|\Delta(\operatorname{Im} y) \square F\|_{L_{\nu}^{p, q}}
$$

where $p \in\left(p_{0}, p_{1}\right), q \in\left(q_{0}, q_{1}\right), \nu>n / r-1$ where $\square$ is so-called generalized wave operator (see [6]), for some fixed $p_{0}, p_{1}, q_{0}, q_{1}$ numbers.

Hence results of previous section can be related to such type estimates but on $L_{\nu}^{p, q}\left(T_{\Omega}^{m}\right)$ and $A_{\nu}^{p, q}\left(T_{\Omega}^{m}\right)$ type spaces on product domains which are based on norms

$$
\left(\int_{V_{1}} \cdots \int_{V_{1}}\left(\int_{V} \cdots \int_{V}\left|F\left(z_{1}, \ldots, z_{m}\right)\right|^{p} d x_{1} \ldots d x_{m}\right)^{\frac{q}{p}} \prod_{j=1}^{m} \Delta^{\nu_{j}-\frac{n}{r}}\left(y_{j}\right) d y_{j}\right)^{\frac{1}{q}}
$$

or

$$
\int_{V_{1}}\left(\int_{V}\left(\cdots \int_{V_{1}}\left(\int_{V}\left|f\left(z_{1}, \ldots, z_{m}\right)\right|^{p_{1}} d x_{1}\right)^{q_{1} / p_{1}}\right) \times \cdots \times \Delta^{\nu_{m}-n / r}\left(y_{m}\right) d y_{m}\right)^{\frac{1}{q_{m}}},
$$

$p_{i}, q_{i} \geq 1, \nu_{j}>n / r-1, j=1, \ldots, m$, where $V_{1}$ or $V$ is equal to $R^{n}$ or $\Omega$.

We can finally define new mixed norm $A_{\alpha}^{p, q}\left(T_{\Omega}^{m}\right)$ spaces on products of $T_{\Omega}^{m}$ domains in two natural ways. Some results previous section can be extended to such spaces. We define analytic spaces with norms in $H\left(T_{\Omega}^{m}\right), m \geq 1$ as follows

$$
A_{\vec{\gamma}}^{\vec{p}, \vec{q}}\left(T_{\Omega}^{m}\right)=\left\{f \in H\left(T_{\Omega}^{m}\right):\left(\int _ { \Omega } \left(\int _ { R ^ { n } } \ldots \left(\int_{\Omega}\left(\int_{R^{n}}\left|f\left(z_{1}, \ldots, z_{m}\right)\right|^{p_{1}} d x_{1}\right)^{\frac{q_{1}}{p_{1}}}\right.\right.\right.\right.
$$




$$
\left.\left.\left.\left.\times \Delta^{\gamma_{1}-\frac{n}{r}}\left(y_{1}\right) d y_{1}\right)^{\frac{p_{2}}{q_{1}}} \times \cdots \times \Delta^{\gamma_{m}-\frac{n}{r}}\left(y_{m}\right)\right) d y_{m}\right)<\infty\right\}
$$

where $1 \leq p_{j}, q_{j}<\infty, \gamma_{j}>\frac{n}{r}-1, i=1, \ldots, m, z_{j}=x_{j}+i y_{j}, j=1, \ldots, m$ and

$$
\begin{aligned}
\tilde{A}_{\vec{\gamma}}^{p, q}\left(T_{\Omega}^{m}\right)= & \left\{f \in H\left(T_{\Omega}^{m}\right): \int_{\Omega^{m}}(\underbrace{\int_{R^{n}} \cdots \int_{R^{n}}}_{m}\left|f\left(z_{1}, \ldots, z_{m}\right)\right|^{p} d x_{1} \ldots d x_{m})^{\frac{q}{p}}\right. \\
& \left.\times \prod_{j=1}^{m} \Delta^{\gamma_{j}-\frac{n}{r}}\left(y_{j}\right) d y_{j}<\infty\right\},
\end{aligned}
$$

where $1<=q, p<\infty, \gamma_{j}>\frac{n}{r}-1, z_{j}=x_{j}+i y_{j}, j=1, \ldots, m$.

Note for particular values of parameters we get spaces studied in [23,24] and in this paper $\left(m=1\right.$ or $p=q$ or $\left.p_{j}=q_{j}=s, \gamma_{j}=\gamma, j=1, \ldots, m\right)$. It can be shown that all these new scales of analytic spaces are Banach spaces. We finally remark the study of these scales of analytic spaces on products of tube domains and related spaces on semiproducts of tube domains is a large interesting and separate problem.

We finally provide a sharp embedding theorem for multifunctional analytic spaces in tubular domains over symmetric cones. The proof repeats the ideas used in proofs of previous theorems and we also follow arguments of the parallel result of Shamoyan-Li (see [17]) in the unit ball. The key ingredient is the estimate from below of Bergman kernel on Bergman ball obtained Recently by Sehba and Nana (see [19]). We need some lemmas.

In the following lemma we denote by $B$ the unweighted Bergman kernel $(B(z, w)=$ $\left.B_{0}(z, w)\right)$.

Lemma 5.1 ([5], Theorem 1.1). For every $\delta>0$, there is a constant $C_{\delta}>0$ such that

$$
\left|\frac{B(\zeta, z)}{B(\zeta, w)}-1\right| \leq C_{\delta} d(z, w)
$$

for all $\zeta, z, w \in T_{\Omega}$, with $d(z, w) \leq \delta$.

For $\nu>\frac{n}{r}-1$ and $w \in T_{\Omega}$, for simplicity, let us consider the normalized reproducing kernel

$$
k_{\nu}(\cdot, w)=\frac{B_{\nu}(\cdot, w)}{\left\|B_{\nu}(\cdot, w)\right\|_{2, \nu}}=\Delta^{-\nu-\frac{n}{r}}\left(\frac{\cdot-\bar{w}}{i}\right) \Delta^{\frac{1}{2}\left(\nu+\frac{n}{r}\right)}(\operatorname{Im} w) .
$$

For $z \in T_{\Omega}$, we define

$$
\tilde{\mu}(z):=\int_{T_{\Omega}}\left|k_{\nu}(z, w)\right|^{2} d \mu(w)
$$


and for $\delta \in(0,1)$ we define the average of the positive measure $\mu$ at $z$ by

$$
\hat{\mu}(z)=\frac{\mu\left(B_{T_{\Omega}}(z, \delta)\right)}{v_{\nu}\left(B_{T_{\Omega}}(z, \delta)\right)} .
$$

The following lemma is a straightforward consequence of [2, Corollary 3.4] and Lemma 5.1.

Lemma 5.2 (see [19]). Let $\nu>\frac{n}{r}-1, \delta>0$ and $z, w \in T_{\Omega}$. There is a positive constant $C_{\delta}$ such that for all $z \in B_{T_{\Omega}}(w, \delta)$,

$$
v_{\nu}\left(B_{T_{\Omega}}(w, \delta)\right)\left|k_{\nu}(z, w)\right|^{2} \leq C_{\delta} .
$$

If $\delta$ is sufficiently small, then there is $C>0$ such that for all $z \in B_{T_{\Omega}}(w, \delta)$,

$$
v_{\nu}\left(B_{T_{\Omega}}(w, \delta)\right)\left|k_{\nu}(z, w)\right|^{2} \geq(1-C \delta) .
$$

The following two results are direct consequences of the above lemma.

Lemma 5.3 (see [19]). Let $\delta \in(0,1)$. Then there exists a constant $C=C_{\delta}>0$ such that

for any $z \in T_{\Omega}$.

$$
\hat{\mu}_{\delta}(z) \leq C_{\delta} \tilde{\mu}(z)
$$

Lemma 5.4 (see [19]). Let $\nu>\frac{n}{r}-1, \delta, t \in(0,1)$. Let $0<\delta_{1}, \delta_{2}, \delta_{3}<\delta$ with $t<\delta_{1} / \delta_{2}<t^{-1}$. Then there exists a constant $C=C(\delta, t)>0$ such that for any $z, w \in T_{\Omega}$ such that $w \in B_{T_{\Omega}}\left(z, \delta_{3}\right)$,

$$
\frac{1}{C}<\frac{v_{\nu}\left(B_{T_{\Omega}}\left(z, \delta_{1}\right)\right)}{v_{\nu}\left(B_{T_{\Omega}}\left(w, \delta_{2}\right)\right)}<C .
$$

We have the following sharp result based on these lemmas.

Theorem 5.4. Let $\mu$ be a positive Borel measure on $T_{\Omega}$ and let $\left\{a_{k}\right\}$ be a sequence an $r$-lattice based on Bergman balls. Let $f_{j} \in H\left(T_{\Omega}\right), j=1, \ldots, m, 0<p_{i}, q_{i}<\infty$, $\alpha>-1, i=1, \ldots, m$. If

$$
\int_{B_{T_{\Omega}}\left(a_{k}\right)} d \mu(z) \leq c \Delta\left(\operatorname{Im} a_{k}\right)^{m\left(2 \frac{n}{r}+\alpha\right)},
$$

then we have the following estimate

$$
\int_{T_{\Omega}} \prod_{i=1}^{m}\left|f_{i}(z)\right|^{p_{i}} d \mu(z) \leq c\left[\prod_{i=1}^{m}\left(\sum_{k=1}^{\infty}\left(\int_{B_{T_{\Omega}}\left(a_{k}\right)}\left|f_{i}(z)\right|^{p_{i}} \Delta(\operatorname{Im} z)^{\alpha} d v(z)\right)^{q_{i}}\right)^{\frac{1}{q_{i}}}\right] .
$$

Moreover the reverse assertion is also valid.

Acknowledgements. This work was supported by the Russian Foundation for Basic Research, grant 13-01-97508. 


\section{REFERENCES}

[1] M. Arsenovic and R. Shamoyan, On multifunctional harmonic functional spaces, Math. Montisnigri 25 (2012), 177-199.

[2] D. Bekolle, A. Bonami, G. Garrigos, C. Nana, M. Peloso and F. Ricci, Lecture notes on Bergman projectors in tube domain over cones, an analytic and geometric viewpoint, Proceeding of the International Workshop on Classical Analysis 2 (2001), 1-75.

[3] D. Bekolle, A. Bonami, G. Garrigos and F. Ricci, Littlewood-Paley decomposition and Besov spaces related to symmetric cones and Bergman projections in tube domains, Proc. Lond. Math. Soc. 2 (2004), 317-360.

[4] D. Bekolle, A. Bonami, G. Garrigos, F. Ricci and B. Sehba, Analytic Besov spaces and Hardy type inequalities in tube domains over symmetric cones, Jour. Fur. Reine und Ang. (2010), 25-56.

[5] D. Bekolle, H. Ishi and C. Nana, Koranyi's lemma for homogeneous Siegel domains of type II, applications and extended results, Bull. Aust. Math. Soc. 90 (2014), 77-89.

[6] A. Bonami, Three related problems of Bergman spaces of tube domains over symmetric cones, Atti Accad. Naz. Lincei Rend. Lincei Mat. Appl. 13 (2002), 183-197.

[7] Y. Chang and R. Fefferman, Some recent developments in fourier analysis and $h^{p}$ theory on product domains, Bull. Amer. Math. Soc. 1 (1985), 1-43.

[8] J. Cima and P. Mercer, Composition operators between Bergman spaces on convex domains in $\mathbb{C}^{n}$, J. Operator Theory 33 (1992), 363-369.

[9] E. Dariush, The d-neumann problem on product domains in $c^{n}$, Math. Ann. 4 (2007), 797-816.

[10] C. Debraj, Spectrum of the complex Laplacian on product domains, Proc. Amer. Math. Soc. 9 (2010), 3187-3202.

[11] C. Debraj and S. Mei-Chi, The Cauchy-Riemann equations on product domains, Math. Ann. 4 (2011), 977-998.

[12] P. Duren and A. Schuster, Bergman spaces, Bull. Amer. Math. Soc. (N.S.) 2 (2005), 241-256.

[13] J. Faraut and A. Koranyi, Analysis on symmetric cones, Clarendon Press, 1994.

[14] P. Jakobczak, The boundary regularity of the solution of the df equation in products of strictly pseudoconvex domains, Pacific J. Math. 2 (1986), 371-386.

[15] T. Jimb and A. Sakai, Interpolation manifolds for products of strictly pseudoconvex domains, Complex Variables, Theory and Application: An International Journal 8 (1987), 222-341.

[16] H. Li, BMO, VMO and Hankel operators on the Bergman spaces of strongly pseudoconvex domains, J. Funct. Anal. 106 (1992), 375-408.

[17] S. Li and R. Shamoyan, On some estimates and Carleson type measure for multifunctional holomorphic spaces in the unit ball, Bull. Sci. Math. 2 (2010), 144-154.

[18] J. McNeal and E. Stein, Mapping properties of the Bergman projection on convex domains of finite type, Duke Math. J. 73 (1994), 177-199.

[19] C. Nana and B. Sehba, Carleson embeddings and two operators on Bergman spaces of tube domains over symmetric cones, Integral Equations and Operator Theory 83 (2015), 151-178.

[20] J. Ortega and J. Fabrega, Mixed norm spaces and interpolation, Studia Math. 109 (1994), 1-43.

[21] W. Rudin, Theory of functions in the unit polydisc, Benjamin, 1969.

[22] W. Rudin, Function theory in the unit ball of $\mathbb{C}^{n}$, Springer-Verlag, 1980.

[23] F. Segba, Hankel operators on Bergman spaces of tube domains over symmetric cones, Integral Equations Operator Theory (2008), 233-245.

[24] F. Segba, Bergman type operators in tube domains over symmetric cones, Proc. Edinb. Math. Soc. (2) 52 (2009), 529-544.

[25] F. Shamoyan and A. Djrbashian, Topics in the theory of $A_{\alpha}^{p}$ spaces, Teubner Texte zur Mathematics 105 (1988).

[26] R. Shamoyan and O. Mihic, In search of traces of some holomorphic spaces on polyballs, Revista notas de matematica 4 (2008), 1-23. 
[27] R. Shamoyan and O. Mihic, Analytic Classes on Subframe and Expanded Disk and the $\mathbb{R}^{s}$ Differential Operator in Polydisk, J. Inequal. Appl. 2009 (2009), Article ID 353801.

[28] R. Shamoyan and O. Mihić, On traces of analytic functions in polyballs, Appl. Anal. Discrete Math. 3 (2009), 198-211.

[29] R. Shamoyan and O. Mihić, On Traces in Some Analytic Spaces in Bounded Strictly Pseudoconvex Domains, 2015 (2015), Article ID 265245.

[30] R. Shamoyan and E. Povprits, Sharp theorems on traces in Bergman type spaces in tubular domains over symmetric cones, Journal Siberian Federal University 6 (2013), 527-538.

[31] M. Vergne and H. Rossi, Analytic continuation of the holomorphic series, Acta Math. 136 (1976), 1-59.

[32] W. Xu, Distances from Bloch functions to some Möbius invariant function spaces in the unit ball of $c^{n}$, Journal of Function Spaces and Applications 7 (2009), 91-104.

[33] K. Zhu, Spaces of Holomorphic Functions in the Unit Ball, Graduate Text in Mathematics 226, Springer, 2005.

${ }^{1}$ BRJANSK STATE UNIVERSITY

241036, BejICKAyA, 79, Bryansk, Russia.

E-mail address: SergKurilenko@gmail.com, rsham@mail.ru 